\title{
A Social Inference Model of Idealization and Devaluation
}

Giles W. Story ${ }^{1,2}$, Ryan Smith $^{3}$, Michael Moutoussis ${ }^{4,2}$, Isabel M. Berwian ${ }^{5}$, Tobias Nolte $^{6,7}$, Edda Bilek ${ }^{4}$, and Raymond J. Dolan ${ }^{2}$

1 Division of Psychiatry, University College London

2 Max Planck-UCL Centre for Computational Psychiatry and Ageing Research, University College London

3 Laureate Insitute for Brain Research

4 Wellcome Centre for Human Neuroimaging, University College London 5 Princeton Neuroscience Institute, Princeton University

6 Wellcome Centre for Human Neuroimaging, University College London 7 Anna Freud National Centre for Children and Families

\section{Author Note}

We have no known conflict of interest to disclose. This work was supported by the Wellcome Trust [Ray Dolan Senior Investigator Award 098362/Z/12/Z], the National Institute for Health Research, and the Max Plank Society. The Wellcome Trust Centre for Neuroimaging is supported by core funding from the Wellcome Trust 091593/Z/10/Z. Tobias Nolte is supported by a Wellcome Trust Research Fellowship to Read Montague. The funders had no role in study design, data collection and analysis, decision to publish, or preparation of the manuscript. Corresponding author: Dr G. W. Story, Max Planck-UCL Centre for Computational Psychiatry and Ageing Research, University College London, Russell Square House, 10-12 Russell Square, London, WC1B 5EH, United Kingdom. Email: 


\begin{abstract}
People often form polarized beliefs about others. In a clinical setting this is referred to as a dichotomous or 'split' representation of others, whereby others are not imbued with possessing mixtures of opposing properties. Here, we formalise these accounts as an oversimplified categorical model of others' internal, intentional, states. We show how a resulting idealization and devaluation of others can be stabilized by attributing unexpected behaviour to fictive external factors. For example, under idealization, less-than-perfect behaviour is attributed to unfavourable external conditions, thereby maintaining belief in the other's goodness. This feature of the model accounts for how extreme beliefs are buffered against counter-evidence, while at the same time being prone to precipitous changes of polarity. Equivalent inference applied to the self creates an oscillation between selfaggrandizement and self-deprecation, capturing oscillatory relational and affective dynamics. Notably, such oscillatory dynamics arise out of the Bayesian nature of the model, wherein a subject arrives at the most plausible explanation for their observations, given their current expectations. Thus, the model we present accounts for aspects of splitting that appear 'defensive', without the need to postulate a specific defensive intention. By contrast, we associate psychological health with a fine-grained representation of internal states, constrained by an integrated prior, corresponding to notions of 'character'. Finally, the model predicts that extreme appraisals of self or other are associated with causal attribution errors.
\end{abstract}

Keywords: splitting, dichotomous thinking, idealization, attribution error, social inference, borderline personality, personality disorder 
People sometimes form polarized beliefs about themselves and others. Thus, others can be idealized, and considered to have exceptionally positive qualities that they do not in reality possess, or devalued, and considered to have unrealistically negative qualities (Beck et al., 2015; Hinshelwood, 1989). Polarized views of self or others feature in several mental health conditions and personality dispositions. For example, an oscillation between idealization and devaluation of others is a feature of borderline personality disorder (BPD) (American Psychiatric Association, 2013; World Health Organization, 1992; World Health Organization, 2018). Similar dynamics, applied to the self, feature as narcissistic personality traits, where "exaggerated self-appraisal may be inflated or deflated, or vacillate between extremes" (American Psychiatric Association, 2013). By contrast, people with paranoid personality focus on extreme negative appraisals of others, manifest as a tendency to misconstrue the neutral or friendly actions of others as hostile, and to form "unsubstantiated 'conspiratorial' explanations of events” (World Health Organization, 1992).

Theories derived from psychoanalysis propose that idealization and devaluation imply polarization in a person's internal model of self or others, referred to as splitting (Akhtar \& Byrne, 1983; Fairbairn, 1940; Kernberg, 1967; Kernberg, 1985; Klein, 1946; Kohut, 1966). A related, and more general, concept in cognitive theory is dichotomous thinking (Arntz \& Veen, 2001; Beck et al., 2015; Napolitano \& McKay, 2007; Veen \& Arntz, 2000), which entails a tendency "to evaluate experiences in terms of mutually exclusive categories rather than to see experiences as falling along continua" (Veen, 2000, p23). Here we adopt the simpler term, splitting, although there is much overlap between the two concepts.

\section{Splitting in Object Relations Theory}


Splitting gained prominence in psychoanalytic thinking with the development of socalled object-relations theory (Gomez, 1997). Here 'object' is used in the sense of 'subject' and 'object', and refers to anything to which a person forms a relational attachment. Accordingly, this branch of psychoanalysis is concerned with how people represent their social relationships. In object-relations theory, splitting is classically seen as an early developmental mechanism by which an infant structures its experience by discriminating between positive and negative affect (Gomez, 1997; Hinshelwood, 1989; Klein, 1946; Zepf, 2012). According to theory, in early development (the first year of life), an infant lacks a representation of self or other as enduring circumscribed entities, with a mixture of properties, and instead experiences disconnected states of extreme satisfaction and frustration (Hinshelwood, 1989; Klein, 1946; Steiner, 1992).

Splitting is also considered a psychological defense mechanism, serving to reduce anxiety by separating sources of security and threat (Feldman, 1992; Hinshelwood, 1989; Kernberg, 1967; Kernberg, 1985; Klein, 1946; Kohut, 1966). This disposition is enhanced by attributing feelings of aggression or frustration to others, who in turn come to be experienced as actively hostile. Thus, the infant is said to experience its own aggression as dangerous, and therefore defensively 'projects' aggressive feelings onto others (Feldman, 1992; Klein, 1946). Consequently, a relationship to another that is experienced as entirely hateful may be the basis of devaluation. Conversely, a child might also attribute feelings of love to others, who are experienced as entirely loving, an arrangement which forms the basis of idealization (Steiner, 1992).

Object-relations theory describes how split representations mature through increasing integration as development proceeds. Fragmented aspects of caregiving experience are gradually brought together to form representations of self and other as whole entities, with a mixture of good and bad qualities (Steiner, 1992; Winnicott, 1945). Such integration is thought to help a child to manage frustration, and to form relationships. For example, representing a parent with a mixture of emotional qualities ensures that their bad aspects are buffered by an expectation of the good. This more integrated representation is associated with 
a stage of 'object constancy', wherein the child's bond with its caregiver becomes a 'stable and enduring inner relation independent of need-satisfaction' (Akhtar, 1994, p441; Freud, 1965; Hartmann, 1952; Mahler et al., 1975).

\section{Splitting as Emotional Concept Learning}

Psychoanalytic theories, such as those described above, are derived inductively from clinical material and, at best, draw on metaphorical constructs (such as ego, libido or internal objects) whose biological and behavioural referents are not defined (see Target \& Fonagy, 1996; Zepf, 2012). Similarly, accounts of splitting in infant development are difficult to verify, owing to the challenges of accessing early infantile emotional experiences. Empirical studies of how older children acquire emotional concepts suggest a broadly similar trajectory (Barrett, 2006; Hoemann et al., 2020). Thus, pre-school children first discriminate positive from negative affect, before learning to differentiate emotions of the same valence, such as anger, sadness or fear (Widen \& Russell, 2003, 2008). Furthermore, children progress from describing emotion in all-or-nothing terms (e.g. as a friend's behavior making them completely angry, sad, or jealous), to recognising grades and mixtures of emotion (Harter \& Buddin, 1987; Harter \& Whitesell, 1989; Westen, 1991; Whitesell \& Harter, 1989). Harter and Buddin (1987) propose that a tendency to process emotion in an all-or-nothing manner corresponds to the Piagetian pre-operational stage of child development, wherein children below the age of around seven years tend to focus on one aspect or dimension of an object at a time, and make few gradations along this dimension (Block, 1982; Piaget, 2013; Piaget \& Cook, 1954). The authors found that a capacity to declaratively express opposite-valence emotions towards a single target at the same time only developed at the age of 10 to 11 years, suggesting that pre-operational children have difficulty integrating emotional dimensions as well as physical ones.

Taken together, object-relations accounts and studies of emotional concept acquisition both suggest that a developing child first recognizes a coarse separation between 
positive and negative affect, consistent with 'splitting'. While object relations theory focuses on the subsequent integration of these 'bad' and 'good' aspects of self and others, studies of emotional development also describe how a child acquires an increasingly differentiated and graded set of emotional concepts. Both accounts describe a child's increasing capacity to represent mixtures of emotion associated with a single object.

\section{Idealization and Devaluation}

Theorists from various fields of psychology have proposed that poorly integrated internal models of self and other result in unstable self-esteem and volatile relationships in adulthood (Bender \& Skodol, 2007; Fairbairn, 1952; Kernberg, 1967; Kernberg, 1985; Kohut, 1966, 2013). From a psychoanalytic perspective, Kernberg suggested that split images of self and other are central to the pathology of BPD. Kernberg proposed that 'borderline personality organization' derives from heightened aggressive impulses, which are either innate, or secondary to excessive frustration during development, and which threaten to annihilate more benign internal images of the self and others (Kernberg, 1967; Kernberg, 1985). Profound splitting is putatively necessary to preserve a feeling of security in the face of these internal threats, by keeping apart good and bad aspects of self and others (Kernberg, 1967). Thus, according to Kernberg, a polarized and fragmented view of others as either 'all-good' or 'allbad', a remnant of incomplete integration during development, leads to unstable relationships in adulthood, characterized by shifts between idealization and devaluation. Horowitz (2004) elaborates a similar idea from the perspective of interpersonal psychology, proposing that, due to inconsistent or abusive parenting, people with BPD have difficulty integrating good and bad attributes of their early caregivers, and as a consequence in later life they are prone to vacillate between assessments of people close to them as either 'all good' or 'all bad'.

Cognitive theories similarly emphasize how dichotomous reasoning, shaped by traumatic early life experience, can underpin beliefs about self and others in adulthood (Beck et al., 2001; Beck et al., 2015; Pretzer \& Beck, 1996). In cognitive approaches, the relational and affective instability that characterise BPD are seen as arising from dysfunctional schemas 
of rejection, abandonment or unlovability, which can be expressed as all-or-nothing logical statements. For example, a person with BPD might believe "If I trust someone I'll be abused or abandoned" (Beck et al., 2015). When these beliefs are activated, people with BPD are prone to view others in extreme and excessively simplistic ways (Arntz et al., 1999; Bhar et al., 2008; Butler et al., 2002; Del Pozo et al., 2018; Geiger et al., 2014). Similar schemas are thought to apply to the self in narcissistic personality disorder. For instance, a person with narcissistic personality might assert, "If I am famous, my life will be a complete success. If I am not famous, my life will be a total failure" (Beck et al., 2015). Thus, according to cognitive theory, abrupt switches between idealized or devalued views of self or others can result from activation of underlying schemas with a dichotomous structure.

In support of these ideas, there is evidence that adults diagnosed with personality disorder make dichotomous evaluations of others' emotions in interpersonal situations (for a review see Bender \& Skodol, 2007; Arntz \& Veen, 2001; Kramer et al., 2013; Moritz et al., 2011; Napolitano \& McKay, 2007; Perry et al., 2013; Preißler et al., 2010; Roepke et al., 2013; Sieswerda et al., 2005; Veen \& Arntz, 2000; Zanarini et al., 2009). In a notable study, healthy adults, adults with $\mathrm{BPD}$, and adults with cluster $\mathrm{C}$ personality disorder were asked to evaluate emotions displayed by different characters in film clips (Veen \& Arntz, 2000). Consistent with dichotomous thinking, people with BPD were found to make significantly more extreme emotional evaluations than both sets of controls. Other studies find that dichotomous thinking, rated using a clinical questionnaire, correlates with overall symptom severity in BPD (Moritz et al., 2011) and occurs in the evaluation of positive as well as negative content (Napolitano \& McKay, 2007).

More recent therapeutic approaches situate dichotomous reasoning in BPD within a broader deficit in a capacity to understand and interpret mental states, referred to as mentalizing (Bateman \& Fonagy, 2013; Fonagy \& Bateman, 2008; Nolte et al., 2019). Here, extreme or simplistic appraisals of others are seen as resulting from heightened affect provoked by attachment stress (Agrawal et al., 2004; Bateman \& Fonagy, 2013; Choi-Kain et al., 2009; Fonagy \& Bateman, 2008; Lyons-Ruth et al., 2005; Nolte et al., 2019). Conversely, 
mentalizing is seen as promoting affect regulation, by providing a context in which to appraise affect (Nolte et al., 2019).

\section{Self-serving and Self-debasing Attribution Errors}

The theories outlined above propose that idealization and devaluation arise from activation of polarized internal models of self and others, where these are shaped by early life experience. This raises a question as to why might such polarized views persist in the face of often being inaccurate? Object-relations theory addresses this by suggesting that splitting and projection continue to operate in adulthood as defensive processes (Fairbairn, 1952; Kernberg, 1967; Kernberg, 1985) though the agency or mechanism responsible for these processes is unclear (see Zepf, 2012; Moutoussis et al., 2015). Here we propose an account of this persistence in terms of causal attribution.

Cognitive theory identifies a number of disrupted reasoning processes that can accompany dichotomous thinking, collectively referred to as cognitive distortions (Beck, 1963; Maruna \& Mann, 2006). Influentially, Beck (1963) described how such erroneous reasoning contributes to the aetiology and maintenance of depression. For example, people with depression tend to form negative causal explanations for events ('arbitrary inference'), and tend to minimize their own achievements while magnifying their problems ('minimization and magnification') (Beck, 1963). Later studies identified that people with depression also tend to attribute success to transient, external factors (e.g. luck), while attributing failure to stable, internal factors (e.g. their lack of ability) (Forgas et al., 1990; Seligman et al., 1979). Such negative attributions prevent depressed people from experiencing the self-enhancing effects of their own success, and thereby maintain a devalued view of the self (Abramson et al., 1978).

Conversely, there is widespread evidence that non-depressed people exhibit positive inferential biases (e.g. Nisbett \& Ross, 1980; Ross, 1977). For instance, healthy adults tend to attribute their own successes to stable, internal factors (e.g. their ability), and their failures to 
transient, external forces (e.g. bad luck) (Miller \& Ross, 1975; Zuckerman, 1979). Similarly, people often minimize responsibility for their offensive behaviour by blaming others or external circumstances (Maruna \& Mann, 2006). Although a degree of 'excuse-making' of this kind can be seen as healthy and desirable for enhancing self-esteem (Seligman \& Schulman, 1986; Seligman, 1991), similar inferential processes might contribute to unrealistically inflated self-appraisal and idealization of the self. In support of this idea, people with high self-esteem are more likely to exhibit a self-serving attributional style (Fitch, 1970).

A proposed explanation for self-serving biases is that people are motivated to believe in their own good-fortunes (Zuckerman, 1979; for a contemporary computational review see Bromberg-Martin \& Sharot, 2020). However, a motivational account cannot easily explain a converse pattern of self-debasing attribution seen in depression. A potentially unifying alternative explanation is that attribution errors change direction to align with a person's expectations (see Bradley, 1978; Miller \& Ross, 1975). For example, it would appear plausible for a person with a high opinion of their own ability to attribute success to their own qualities, and failure to external factors. Indeed, in support of an expectation-based theory, self-serving attribution error has been found to correlate with prior expectations about task performance. That is, people who expect to perform well on a task tend to attribute success to internal factors, while people who expect to perform badly tend to attribute success to external factors (Feather, 1969; Feather \& Simon, 1971a, 1971b). Here, we suggest that formally equivalent attributions could also apply to inference about others. Thus, a person with positive expectations of others would tend to attribute others' good behaviour to internal factors (e.g. good character) and their bad behaviour to transient or external factors (e.g. stress or intoxication). By contrast, a person with negative expectations of others would tend to attribute others' bad behaviour to internal factors (e.g. malign intent) and good behaviour to external factors (e.g. outside assistance or an ulterior motive). Importantly, an expectationdriven theory of attribution suggests that shifts between idealization and devaluation ought to be accompanied by corresponding changes in attribution error. However, to our knowledge 
this possibility has not been directly examined. Rather, cognitive approaches have tended to consider dichotomous thinking and attribution error as separate forms of 'cognitive distortion' (Maruna \& Mann, 2006).

Here we introduce a model of idealization and devaluation, which predicts that attribution errors stabilise dichotomous beliefs; this arises of the probabilistic nature of the model, whereby a person arises at the most plausible explanation for their observations, given their current expectations. This feature of the model accounts for how extreme beliefs about self or others are often resistant to counter-evidence, but are nevertheless prone to precipitous changes of polarity. The model captures a computational structure common to both cognitive and psychoanalytic theories, therefore bridging psychiatric and psychotherapeutic concepts across traditions.

\section{A Computational Model of Idealization and Devaluation}

To study idealization and devaluation, we implement a probabilistic model for how other peoples' internal states combine with external circumstances to give rise to their behaviour. In keeping with contemporary approaches (e.g., Barnby et al., 2020; Diaconescu et al., 2020; Moutoussis, Fearon, et al., 2014; Moutoussis, Trujillo-Barreto, et al., 2014; Reiter et al., 2019; Wellstein et al., 2020), we suggest that humans use such a model to infer others' internal states in social interactions. We focus on representations of others, however as we address in the Discussion, the formal principles are easily generalized to beliefs about the self. A key aspect of the model is that others' internal states are expressed in terms of probabilistic hypotheses about the causes of their behaviour.

\section{Bayesian Inference}

Myriad theories in computational neuroscience propose that our brain forms probabilistic hypotheses about the hidden causes of its inputs by approximating Bayesian 
inference (Chater \& Oaksford, 2008; Dunsmoor et al., 2015; Friston et al., 2016, 2017;

Gershman \& Niv, 2020; Gershman, 2017; Gershman et al., 2013; Gershman et al., 2015;

Glimcher, 2004; Noorani \& Carpenter, 2016; Tomov et al., 2018). Bayes' theorem states how new information can be optimally combined with prior knowledge to update belief in particular hypothesis as follows:

$$
p(s \mid o)=\frac{p(s) p(o \mid s)}{p(o)}
$$

Here, $p(s \mid o)$ is the posterior probability of an hypothesis that the world is in state, $s$, given some observations, $o$. A prior probability, $p(s)$ denotes level of belief in an hypothesis before seeing the data. A likelihood term, $p(o \mid s)$ denotes the probability of observing such data, if the world were indeed in state $s$. The denominator, $p(o)$ is a normalizing constant, which ensures the probabilities of alternative hypotheses sum to one. The numerator of Bayes' theorem describes how hidden states give rise to observations, and is therefore referred to as a generative model. In psychological terms this corresponds to an internal model of how the world works. Inference then entails working backwards to discover which hidden (or latent) states best explain the available observations (Griffiths \& Yuille, 2008, p19).

\section{Bayesian Social Inference}

To illustrate how Bayes' rule can be applied to social inference, consider the following scenario. You are due to meet a friend for coffee and they fail to arrive on time. As you wait for your friend to arrive you consider the possibility that they may not want to see you. However, you also consider alternative possibilities, including that they were simply delayed due to unforeseen signalling problems on the metro system. 
Each possibility can be expressed as a random variable: the friend's promptness in arriving $\left(o_{1}\right)$, the transport news $\left(o_{2}\right)$, their motivation to see you $\left(s_{1}\right)$ and metro delays $\left(s_{2}\right)$. These are depicted as nodes in a directed graphical model in Figure 1a. Here, promptness and transport news are observable variables, while reliability and metro delays are hidden states. In this example promptness $\left(o_{1}\right)$ depends on both an intention to be on time $\left(s_{1}\right)$ and metro delays $\left(s_{2}\right)$. Transport news $\left(o_{2}\right)$ by contrast depends only on metro delays $\left(s_{2}\right)$. We can therefore write the joint distribution over observations and hidden states (that is, how likely is any combination of observations and their hidden causes) as follows:

$$
P\left(o_{1}, o_{2}, s_{1}, s_{2}\right)=P\left(o_{1} \mid s_{1}, s_{2}\right) P\left(o_{2} \mid s_{2}\right) P\left(s_{1}\right) P\left(s_{2}\right)
$$

Belief in different hidden states can be updated in light of observations according to Bayes' rule as above. The above arrangement can be further elaborated to express a timedependence of hidden states, creating a Hidden Markov Model (HMM) (Bishop, 2006; Chater \& Oaksford, 2008). Consider, for example, that you meet your friend for coffee every morning, and that their promptness in arriving depends on a combination of their motivational state that morning and the status of the transport network. Here, rather than a graphical model, relationships between hidden states are described in a state transition diagram. A state transition diagram for an HMM based on the example above is shown in Figure 1b. Such a model specifies, in a transition matrix, a propensity for hidden states to change over time; for instance, the model specifies a probability that, if there were transport delays yesterday, there will also be transport delays today.

Finally, as shown in Figure 1c, beliefs can be updated across nested timescales. For instance, one might infer not only a friend's intention each day, but also their prevailing intentions from one week to the next, corresponding to an assessment of their character or personality. This higher-order representation, $s_{3}$, enters the model through a prior over 
starting states at the level below, $p\left(s_{1} \mid s_{3}\right)$, in other words an expectation about the other's internal state. Such an hierarchical organization is consistent with contemporary computational models of human structure learning (Gershman, 2017; Gershman \& Niv, 2012; Gershman et al., 2015; McCormack et al., 2015; Tenenbaum \& Griffiths, 2001; Tomov et al., 2018), which have recently also found application in relation to emotional concept learning (Smith et al., 2018; Smith, Parr, et al., 2019). 
Figure 1

Models of Social Inference

a Social inference

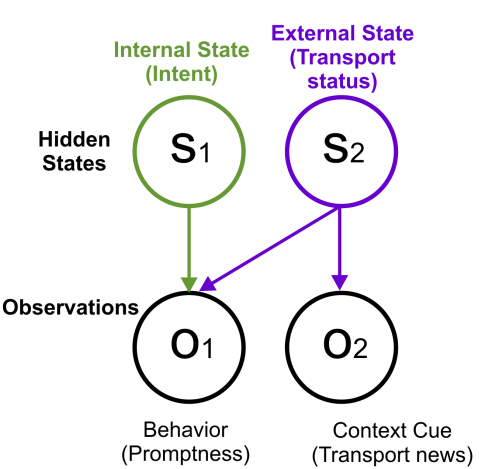

b Dynamic social inference

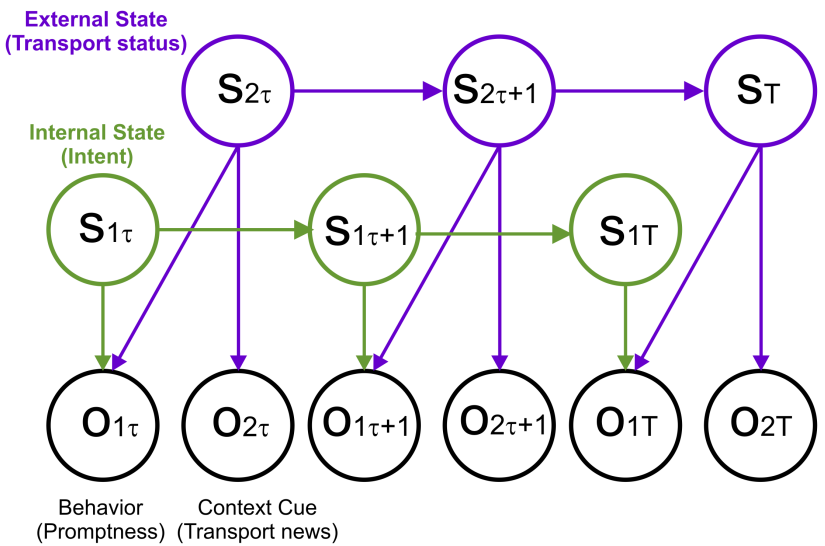

C Hierarchical dynamic social inference

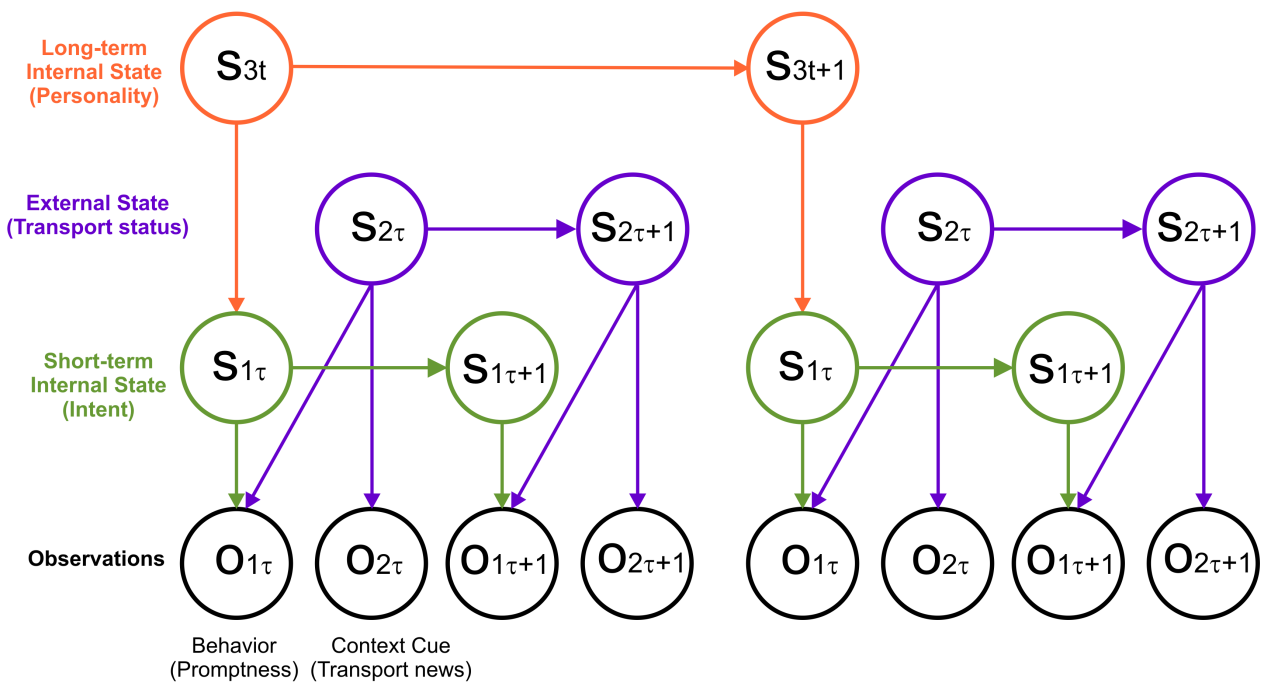

Note. a) Probabilistic graph illustrating how hidden states generate observed

variables. Here another person's behaviour ( $o_{1}$, e.g. promptness in attending an appointment) depends on both their internal state ( $s_{1}$, e.g. motivation to attend) and an external context $\left(s_{2}\right.$, e.g. transport delays). A context cue ( $o_{2}$, e.g. transport news) by contrast depends only on the external context $\left(s_{2}\right)$. b) Hidden Markov Model (HMM) specifying a propensity for hidden states to change over time. c) The state transition diagram is expanded to add a higher-order 
representation, $s_{3}$, corresponding to a prior over internal states, i.e. character or personality, updated over a longer-timescale.

\section{A Probabilistic Model of Splitting}

Here we model social inference based on the above example, by equipping a simulated subject with generative models of the forms shown in Figure $1 \mathrm{~b}$ and $1 \mathrm{c}$. In each case, a subject represents internal and external states, $s_{1}$ and $s_{2}$, respectively corresponding to the intention of another person and the environmental context. These jointly generate two observations: the other person's behaviour, $o_{1}$, and a contextual cue, $o_{2}$. We incorporate an expectation that intentions or circumstances can change over time, formalized in a transition matrix. Both hidden states and observations are expressed as discrete quantities. We operationalize observed behaviour on an ordinal scale from 1 to 10 , representing the objective level of cost or benefit for the subject.

First-Order Internal States. We first consider a generative model of the form shown in Figure 1b. Here, we suggest that a healthy model of others entails a graded representation of internal states $\left(s_{1}\right)$, corresponding to varying degrees of benevolence in another's intentions: $s_{1}=\{$ Bad, Moderately Bad,Neutral, Moderately Good, Good $\}$. These five internal states map onto behaviour in a realistic way, accounting for intermediate observations, $P\left(o_{1} \mid s_{1}\right)$ (Figure 2a).

By contrast, we formalize splitting as a representation of internal state $\left(s_{1}\right)$ in terms of polarized, exclusive categories, rather than as a continuum. Internal states are modelled by just two categories, separated according to affective valence into 'Bad' and 'Good': $s_{1}=\{$ Bad, Good $\}$. 'Bad' and 'Good' states give rise to a narrow range of low- or highvalued behavioural outcomes respectively (Figure $2 b$ ). Such a model inevitably leads to inference about others' mental states that oscillates between extremes. We refer to this arrangement as 'first-order splitting'. 
Second-Order Internal States. We also consider how splitting might occur at a higher representational level, that of 'personality'. Here, we assume that the subject is equipped with an hierarchical model as shown in Figure 1c, with a graded representation of internal states at the first level, $\left(s_{1}\right)$, and consider different forms of prior expectation, $P\left(s_{1} \mid s_{3}\right)$, about others' internal states. A unimodal prior, $s_{3}=\{$ Mixed Person $\}$, priviliges a modal internal state, but also allows other for others, in proportion to their relatedness to the modal state (Figure $2 \mathrm{c}$ ). We configure $P\left(s_{1} \mid s_{3}\right)$ such that others' intentions are expected to start as Neutral, with some uncertainty around this estimate. This arrangement can be seen as arising out of a healthy emotional development, whereby a differentiated representation of emotion $\left(s_{1}\right)$, is balanced by an 'integrated' superordinate representation of another person as a whole $\left(s_{3}\right)$.

On the other hand, a strongly bimodal prior, $s_{3}=\{$ Bad Person, Good Person $\}$, prescribes a strong dominance of either 'Good' or 'Bad' internal states (Figure 2d), and therefore leads to inference that oscillates between poles of idealization and devaluation. Such 'second-order splitting' allows for a possibility that people are capable of detecting intermediate changes in others' mental states, and yet make dichotomous inferences about their overall personality, or disposition. We parameterise second-order splitting as the symmetric separation between the two modes, termed $d^{\prime}$ (the special case where the two modes are equal, $d^{\prime}=0$, entails a unimodal prior). 


\section{Figure 2}

First- and Second-Order Models of Splitting

\section{First-Order Splitting}
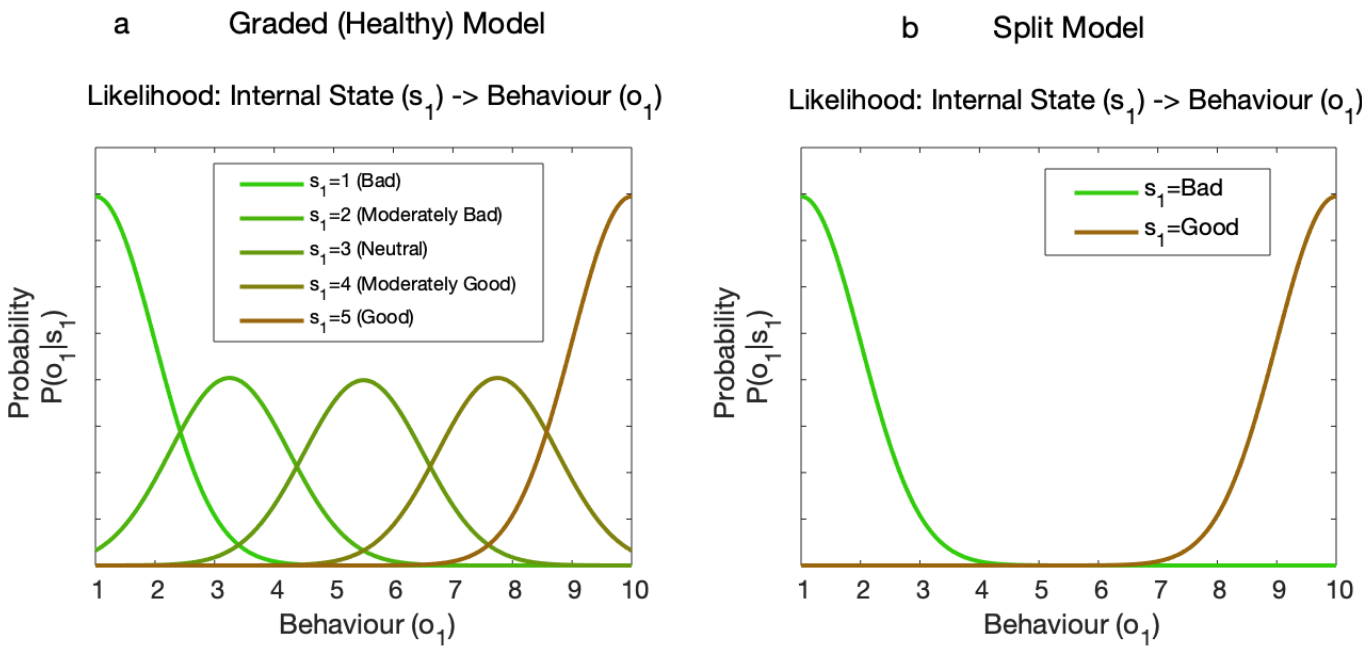

Second-Order Splitting

c Integrated (Healthy) Model

Likelihood: Personality $\left(\mathrm{s}_{3}\right)->$ Internal State $\left(\mathrm{s}_{1}\right)$

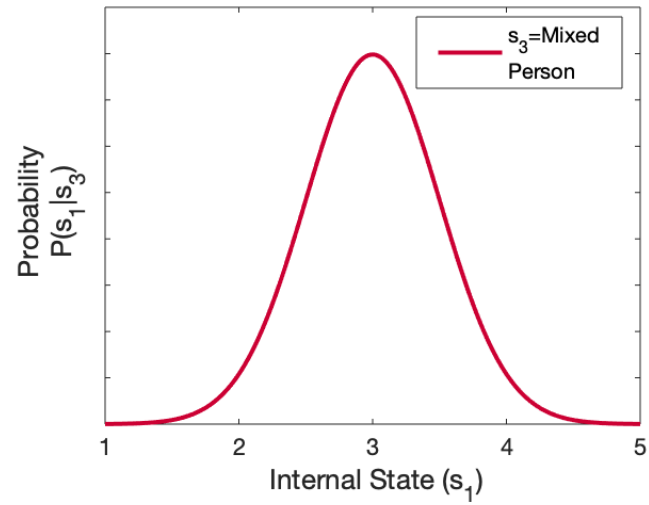

d Split Model

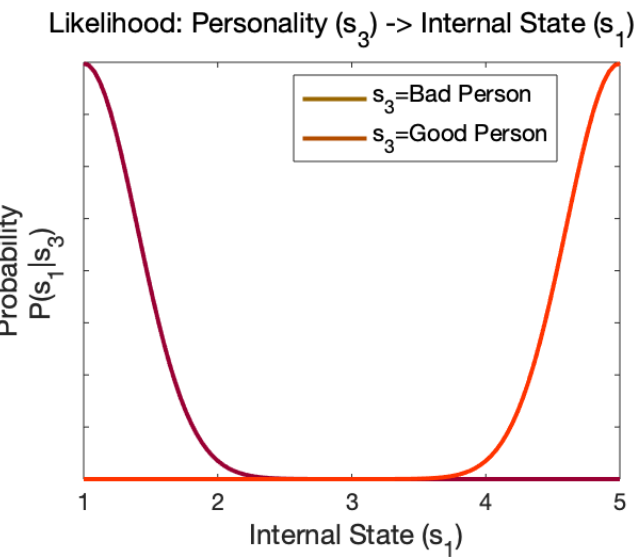

Note. Likelihood distributions are shown for two different forms of splitting. The first row illustrates 'first-order' splitting, over internal states (e.g. intentions or motivations). Each panel shows the subject's model for how the other's internal states $s_{1}$ generate observed behaviour, $o_{1}$ (here external state, $s_{2}$, is assumed to be 'Neutral'). The other's observed behaviour is operationalized on an ordinal scale from 1 to 10 , representing the objective level of benefit to the subject (e.g. the degree of promptness in arriving for an appointment). Although this is a discrete state space model, probability distributions are displayed as smoothed continuous lines for illustrative purposes. a) A graded model consists of five levels 
of internal state b) A split model entails two levels of internal state, Bad and Good. The second row illustrates 'second-order' splitting, over personalities, i.e. prior expectations about the other's internal states at the level below. In each case the subject is equipped with a graded model of internal state at the level below, $s_{1}$, as shown in panel a). c) An 'integrated' model consists of a unimodal prior over internal state d) A split model entails a bimodal prior over internal state.

External States. To model attribution errors, the subject is equipped with an external state dimension, $s_{2}$, representing three degrees of favourability in circumstances: $s_{2}=$ \{Unfavourable, Neutral, Favourable\}. Here, unfavourable external circumstances worsen behaviour $\left(o_{1}\right)$, despite Good intentions, while Favourable circumstances improve behaviour, despite Bad intentions. This is shown for a graded model in Figures 3a and 3b. We consider models in which external factors either give rise to reliable contextual cues $\left(o_{2}\right)$ (Figure 3c), or provide no cue information.

Effects of external state for a (first-level) split model are shown in Figures $4 \mathrm{a}$ and $4 \mathrm{~b}$. Here, Unfavourable conditions generate more variable behaviour when intentions are Good, but have no effect on behaviour when intentions are Bad. This increases the scope of behaviour compatible with Good intentions. Conversely, Favourable conditions generate more variable behaviour when intentions are Bad, but have no effect when intentions are Good. This increases the scope of behaviour compatible with Bad intentions. We show how with this model, in the absence of contextual cues (Figure 4c), phases of idealization and devaluation are stabilized by attributing belief-incongruent observations to fictive external factors. 


\section{Technical Model Specifications}

We operationalize the generative models following a previously published nonparametric Bayesian scheme (Friston et al., 2017; Smith, Parr, et al., 2019; Smith, Schwartenbeck, et al., 2020). Belief in hidden states is updated in light of observations according to Bayes' rule. Generalising, so that $\boldsymbol{o}=\left\{o_{1}, \ldots, o_{n}\right\}$ denotes the set of all observable random variables, and $\boldsymbol{s}=\left\{s_{1} \ldots s_{f}\right\}$ denotes the set of all hidden state factors, we can write:

$$
P(\boldsymbol{s} \mid \boldsymbol{o})=\frac{P(\boldsymbol{s}) P(\boldsymbol{o} \mid \boldsymbol{s})}{P(\boldsymbol{o})}
$$

The likelihood term, $P(\boldsymbol{o} \mid \boldsymbol{s})$, expressing statistical relationships between hidden states and data, can be further specified in terms of a probability distribution, with parameters $\boldsymbol{\theta}$.

An HMM extends the generative model to assume a sequence of $T$ observations, generated by an evolution of hidden states across time. For each hidden state factor, a $K \times K$ transition matrix, $P\left(\boldsymbol{s}_{t} \mid \boldsymbol{s}_{t-1}\right)$, determines the probability that each of $K$ hidden states will follow one another. A generative model (the numerator of Bayes' rule) can then be written as the joint distribution over observations, hidden states and parameters as follows:

$$
P(\widetilde{\boldsymbol{o}}, \tilde{\boldsymbol{s}}, \boldsymbol{\theta})=P\left(\boldsymbol{s}_{\mathbf{0}}\right) P(\boldsymbol{\theta}) \prod_{t=1}^{T} P\left(\boldsymbol{o}_{t} \mid \boldsymbol{s}_{t}\right) P\left(\boldsymbol{s}_{t} \mid \boldsymbol{s}_{t-1}\right)
$$

Where $\widetilde{\boldsymbol{o}}=\left\{\begin{array}{lll}\boldsymbol{o}_{\mathbf{1}} & \ldots & \boldsymbol{o}_{T}\end{array}\right\}$ denotes the sequence of observations, $\widetilde{\boldsymbol{s}}=\left\{\boldsymbol{s}_{\mathbf{1}} \ldots \boldsymbol{s}_{T}\right\}$ denotes the sequence of hidden states over time, and $\boldsymbol{\theta}$ denotes the model parameters. Note that $P\left(\boldsymbol{s}_{\mathbf{0}}\right)$ 
is a prior over the starting states, while the transition matrix acts as a prior over states conditioned on the states at the previous time step.

We define a likelihood term, $P\left(\boldsymbol{o}_{t} \mid \boldsymbol{s}_{t}\right)$, as a matrix, A. Each entry $A_{\boldsymbol{j} \boldsymbol{k}}$ of this matrix represents the probability of given observation $o=j$ being generated when a particular setting of a given hidden state, $s=k$ is active. Specifically, for each hidden state factor at each time point, $P\left(o_{t} \mid s_{t}\right)$ is a multinomial likelihood distribution governing the probability of observing each outcome at each level of the hidden state. We generate the multinomial distribution at each level of hidden state from a discretized Gaussian distribution, with a specified mean and variance (see Figures 3 and 4).

We define a transition probability $P\left(\boldsymbol{s}_{t} \mid \boldsymbol{s}_{t-1}\right)$ as a matrix, $\boldsymbol{B}$. We specify this as a discrete random walk over different levels of each hidden state dimension. The level of noise in the random walk is set to a fixed level, irrespective of the volatility of the true process generating observations. Unless otherwise specified, the level of noise is identical for graded and split models, shown for internal states of a first-level graded model in Figure 3c. For firstlevel split models we also consider a transition matrix with near-zero noise, corresponding to a belief that internal states are high unlikely to change, shown in Figure 4c.

Finally, we define a prior distribution over starting states, $P\left(\boldsymbol{s}_{\mathbf{0}}\right)=P\left(\boldsymbol{s}_{\boldsymbol{t}=\mathbf{0}}\right)$, as a vector, $D$. For first-level models, this is set to a uniform distribution. For hierarchical models, outcomes at the second level correspond to starting internal states at the level below: at each time step, $t$, a likelihood matrix, $P\left(s_{1, \tau=0} \mid s_{3, t}\right)$, specifies a probability distribution over starting internal states, $s_{1}$, for each level of $s_{3}$.

Using notation $\operatorname{Cat}(\mathbf{A})=\operatorname{Pr}(o=j \mid s=k)$, we can write:

$$
\begin{gathered}
P\left(\boldsymbol{o}_{t} \mid \boldsymbol{s}_{t}\right)=\operatorname{Cat}(\boldsymbol{A}) \\
P\left(\boldsymbol{s}_{t} \mid \boldsymbol{s}_{t-1}\right)=\operatorname{Cat}(\boldsymbol{B})
\end{gathered}
$$




$$
P\left(\boldsymbol{s}_{\mathbf{0}}\right)=\operatorname{Cat}(D)
$$

Inference consists of inverting the model to infer the hidden states likely to have generated observations. Model inversion is achieved within a variational Bayesian method, which minimises free energy with respect to expectations about hidden states. The details of this method have been described elsewhere (Friston et al., 2017; Smith et al., 2021), and entail a variational (marginal) message-passing algorithm to arrive at an approximate posterior distribution through a gradient descent on variational free energy (Parr et al., 2019; see Gershman \& Blei, 2012 for a general discussion of inference in non-parametric Bayesian models). Unlike the most common uses of this modelling framework, our model does not include policies for action. The configurations of $\mathbf{A}, \mathbf{B}$ and $D$ are given by Dirichlet distributions, the sufficient statistics, $\boldsymbol{\theta}=\{\mathbf{a}, \mathbf{b}, d\}$, of which are concentration parameters that can be regarded as the number of occurrences encountered in the past, specified as shown in Figures 3 and 4 (Friston et al., 2017; Gershman \& Blei, 2012). 


\section{Figure 3}

Graded (Healthy) Model Specification
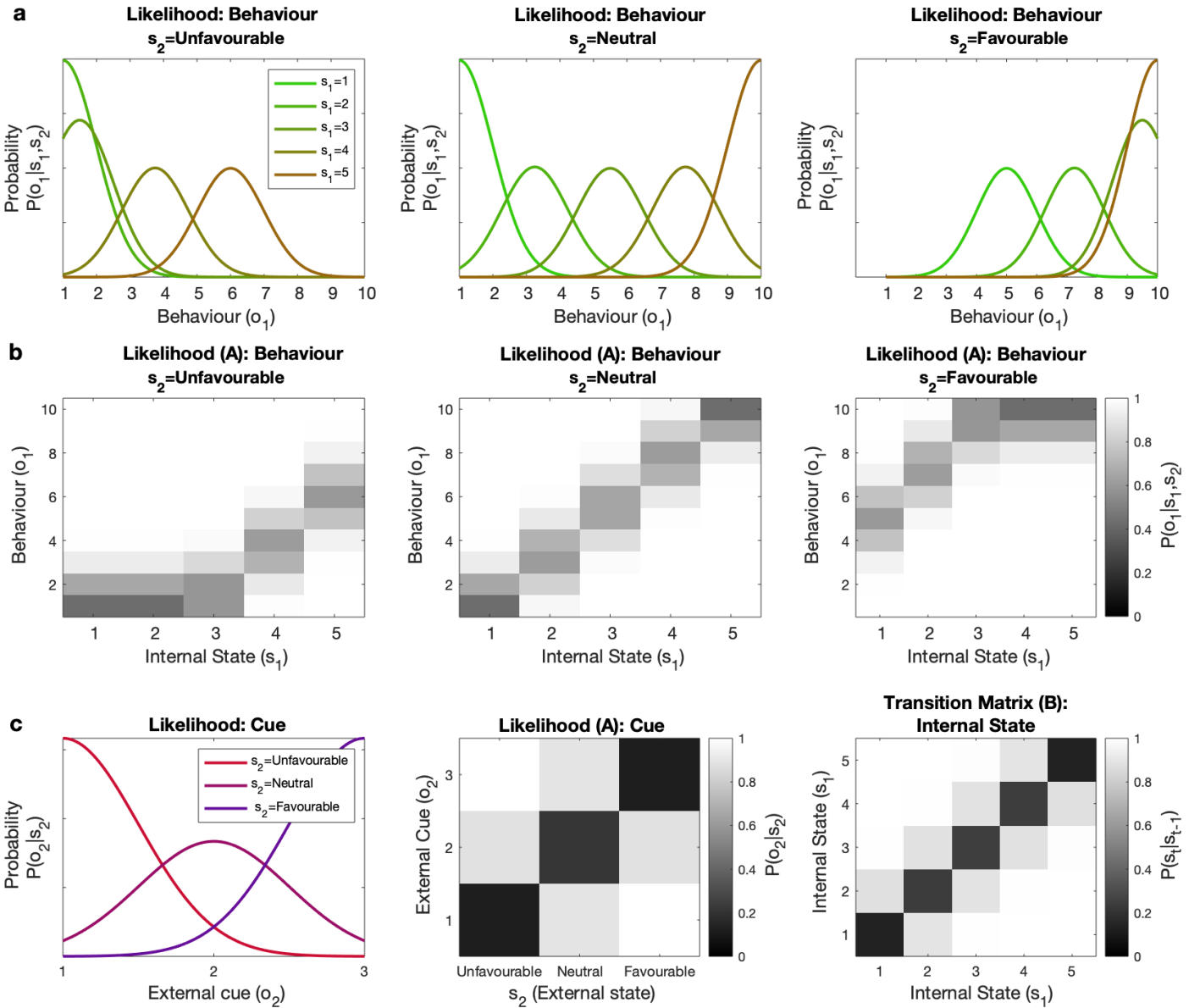

Note. Likelihood and transition matrices for a graded model at the first level. Rows a) and b):

Likelihood distributions illustrating how internal and external hidden states jointly generate observed behaviour. These are displayed both as smoothed probability distributions (a), and as the discrete matrices used in the model (b). An Unfavourable external state worsens behaviour, while a Favourable external state improves behaviour. c) Left two panels: Likelihood distributions illustrating how external hidden states generate cues. As explained in the main text we consider different forms of likelihood for external state cues, either informative (shown here) or uniformative (see Figure 4). Right panel: A transition matrix is specified as a discrete random walk over different levels of each hidden state dimension (shown for internal states). 


\section{Figure 4}

First-Level Split Model Specification
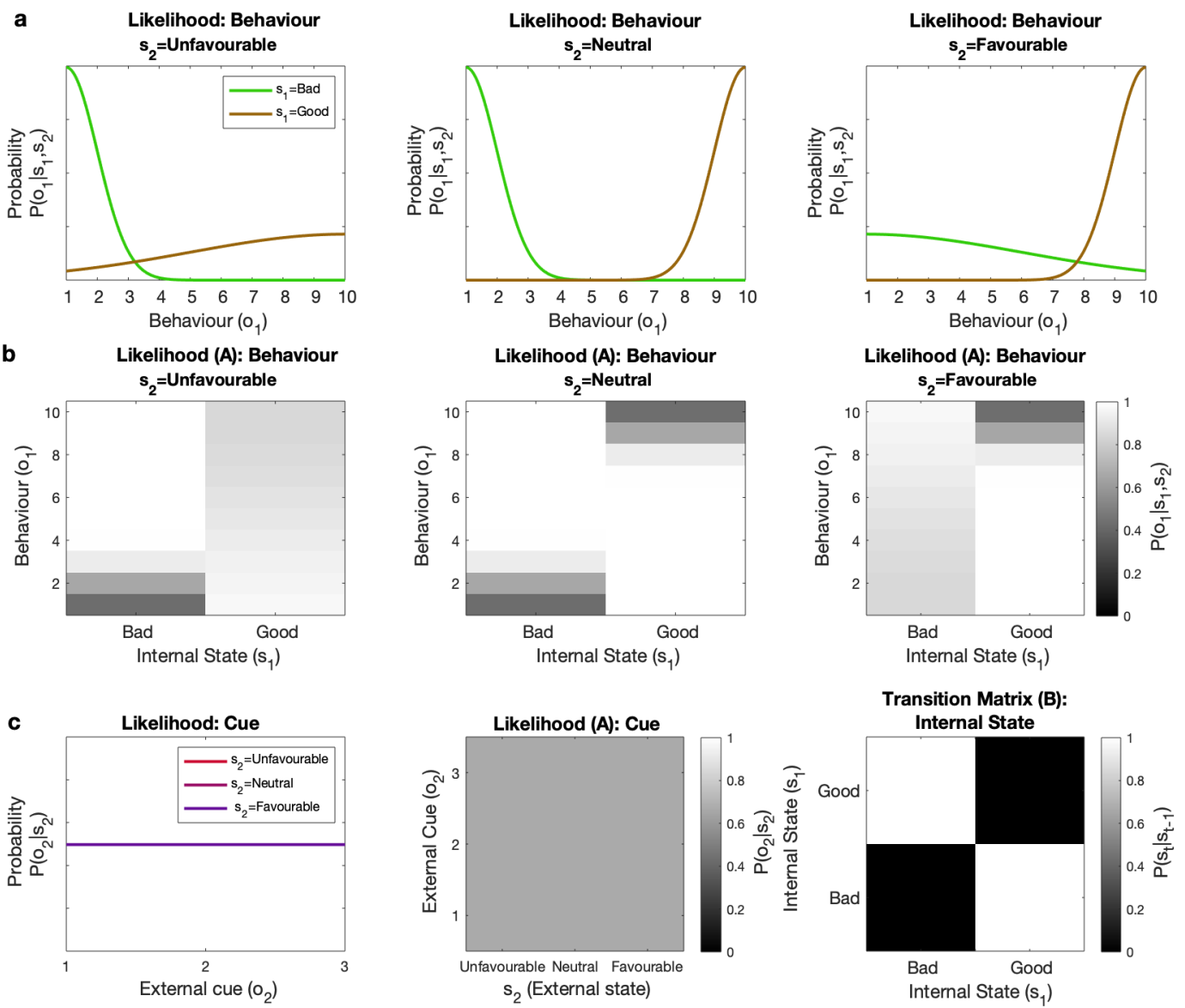

Note. Likelihood and transition matrices for a split model at the first level. Rows a) and b):

Likelihood distributions illustrating how internal and external hidden states jointly generate observed behaviour. These are displayed both as smoothed probability distributions (a), and as the discrete matrices used in the model (b). An Unfavourable external state worsens behaviour, by increasing the scope of behaviour accounted for by Good intentions, corresponding to an 'excuse'. A Favourable external state improves behaviour, by increasing the scope of behaviour accounted for by Bad intentions, corresponding to an 'ulterior motive'. c) Left two panels: Likelihood distributions illustrating how external hidden states generate cues. As explained in the main text we consider different forms of likelihood for external state cues, either informative (see Figure 3) or uniformative (shown here). Right panel: A transition matrix is specified as a discrete random walk over different levels of each hidden state 
dimension (shown for internal states). As explained in the main text we consider different degrees of noise in the internal state transition matrix. Here, we show a transition matrix with a near-zero probability of internal state changes.

\section{Simulation and Trial Order}

We consider inference under the models described above, when provided with changes in another person's observed behaviour over time. Note that the subject's generative model might differ from the true process generating the observations, referred to as the generative process (Friston et al., 2017; Gershman \& Blei, 2012). In each case, we generate behaviour from a graded model. The simulated subject is given a series of observations generated by this process. At each encounter, the subject first observes a cue, $o_{2}$, which gives information regarding the external state (information about transport delays), and subsequently observes the other's behaviour, $o_{1}$ (degree of promptness).

\section{Results}

\section{Graded (Healthy) Social Inference}

We first simulate social inference at the first level (as shown in Figure 1b), with a uniform prior over internal states. With a graded representation of internal states (Figure 2a), the simulated subject smoothly tracks changes in the other's behaviour across time. If the subject is also provided with reliable information about external states, in the form of a contextual cue $\left(\mathrm{O}_{2}\right)$, they accurately infer that changes in behaviour are due to changes in external rather than internal states (Figure 5a). This arises since belief in one hidden state affects belief in another. To appreciate this, consider the 'meeting for coffee' scenario. After observing the friend's lateness, the propositions that they do not want to attend or that there are delays on the transport system both become more likely. Whereas these two possibilities 
were previously independent, after observing the degree of lateness $\left(o_{1}\right)$, they are now conditionally dependent. Thus, after seeing the status of the metro system $\left(o_{2}\right)$ and finding that there are indeed delays, the subject is no longer certain of their friend's bad intent: evidence in support of this is explained away by the transport delays. As a result, beliefs about the other's internal state remain relatively constant, despite their changing behaviour.

We next lesion the generative model so that cues are uninformative to the subject, reducing the precision of the likelihood mapping between external states and cues. The subject must therefore infer hidden states from behaviour alone. As shown in Figures $5 \mathrm{~b}$ and $5 \mathrm{c}$, the subject is still capable of tracking the other's behaviour. However, they are unable to discern whether behaviour results from internal or external factors. As a result, the subject makes attribution errors, attributing external state changes to internal factors and vice versa. Such inference is erroneous with respect to the ground-truth, but Bayes' optimal given available information. This instantiates a previously proposed notion that attribution errors might arise from incomplete contextual information (Miller \& Ross, 1975), and that arbitrary inference is greater in ambiguous scenarios (Beck, 1963). 


\section{Figure 5}

Social Inference of a Graded (Healthy) Model: First-Level

a Context Cue:
Changing External State

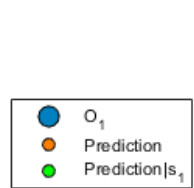

Posterior Prediction $\mathrm{O}_{1}$

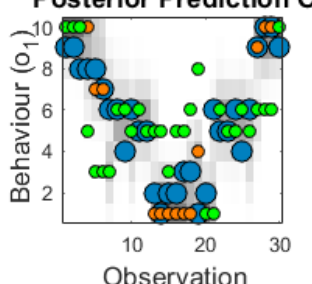

Observation

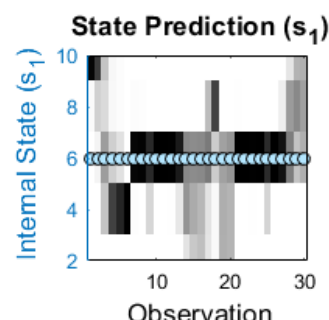

Observation

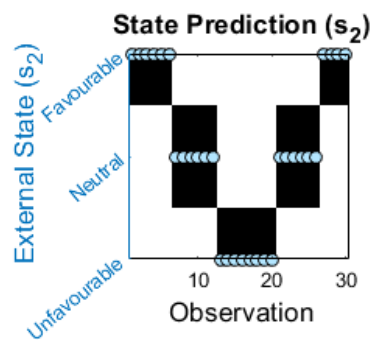

b

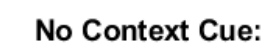

c

No Context Cue:

Changing Internal State
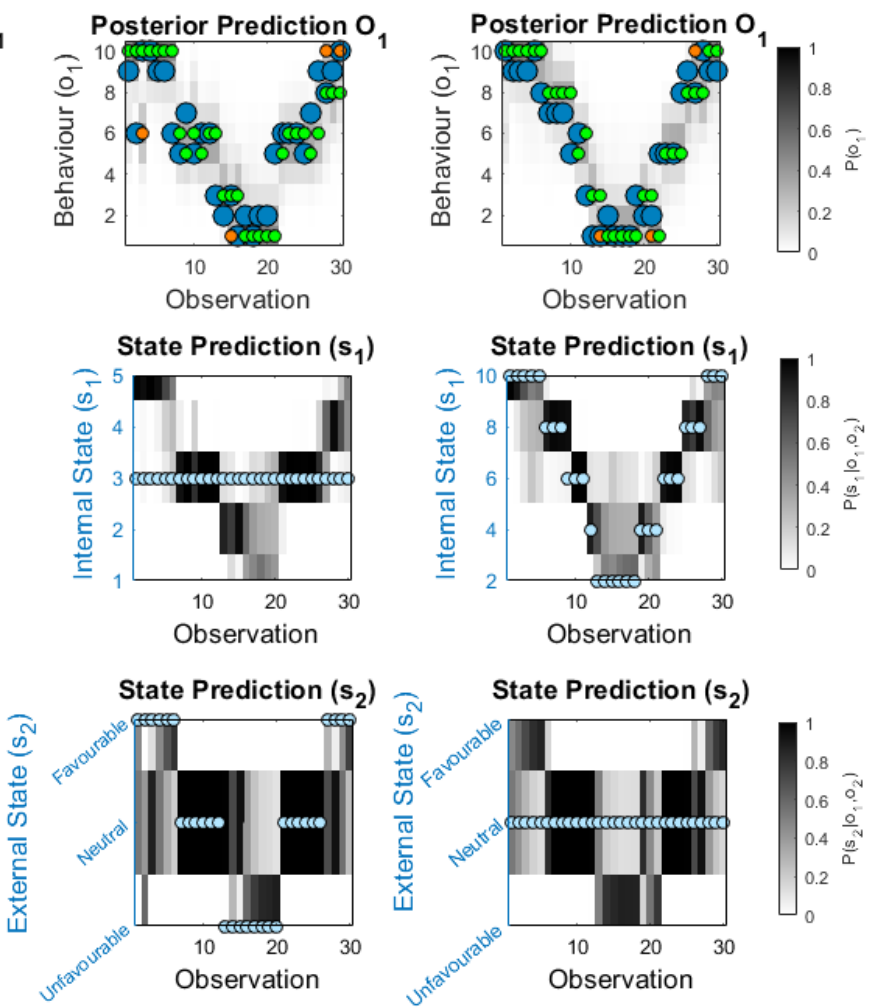

Note. a) The subject observes a cue $\left(o_{2}\right)$ providing reliable information about external state.

Observed behaviour is generated from a graded model (Figure 3) by changing the external state factor. Top panel: observed behaviour (blue circles) gradually worsens, before improving again. The subject accurately predicts changes in behaviour. Maximum $a$ posteriori predictions about upcoming behaviour, after observing the cue, conditioned on inferred internal state, are shown as green circles. Predictions given both internal and external states are shown as orange circles, where these differ from predictions based on internal state alone. Middle panel: True internal states (light blue circles) remain constant. The subject's posterior beliefs over internal states for the most part reflect this. Bottom panel: The subject accurately tracks external state changes. b) With an uninformative external state cue, the subject is unable to discern the causes of behaviour and, in part, attributes external state 
changes to internal factors c) Similarly, without a reliable external state cue, the subject in part attributes internal state changes to external factors.

\section{Split Social Inference: Idealization and Devaluation}

Figure 6 shows social inference under a first-level split model (of the form shown in Figure 3) when confronted with the same pattern of behaviour. As previously, when provided with a cue reliably indicating that the external context has not changed, the subject accurately infers that changes in behaviour result from changes in internal state. Here, although the other's true internal state in fact changes in a graded manner, the subject can only infer either Good or Bad intent (Figure 6a). Hence the subject switches between inferring Good and Bad intent readily and symmetrically.

Phases of idealization and devaluation seen clinically exhibit a degree of stability. We find that these opposite poles become stable in the face of countervailing evidence if the split model is lesioned so that cues are no longer informative (as shown in Figure 4c). Importantly here, the subject persists in inferring Good intent, even after seeing several instances of mediocre behaviour. Only after observing very poor behaviour does the subject switch to infer Bad intent. The subject then persists in inferring Bad intent, even after seeing several instances of good behaviour that would previously have supported a conclusion of Good intent (Figure 6b).

This bistability is associated with a subject's assigning changes in behaviour to changes in the external hidden state dimension. During idealization, instances of less-thanperfect behaviour are attributed to Unfavourable external conditions, $s_{2}$. In other words, the external circumstances are used to excuse behaviour that does not accord with expectations, leaving the other's Good intent untarnished. That is, that given a state of idealization, in the absence of reliable cues, and upon observing relatively poor behaviour, the agent changes their beliefs about external circumstances rather than making a drastic shift from idealization to devaluation. 
Only after observing 'inexcusably bad' behaviour, does the subject switch to inferring Bad intent. Beyond this point, improvements in behaviour are explained away as due to Favourable external conditions, $s_{2}$. We note again that here that Favourable external conditions could equally be conceptualized as an additional internal factor in the form of an ulterior motive. Notably, the subject only switches back to inferring Good intent after seeing extremely good behaviour. This finding resembles an intuition that concrete gestures of 'going the extra mile' can be required to recover relations following ruptures in cooperation (Bateman et al., 2015). 


\section{Figure 6}

Social Inference of a First-Level Split Model: Idealization and Devaluation

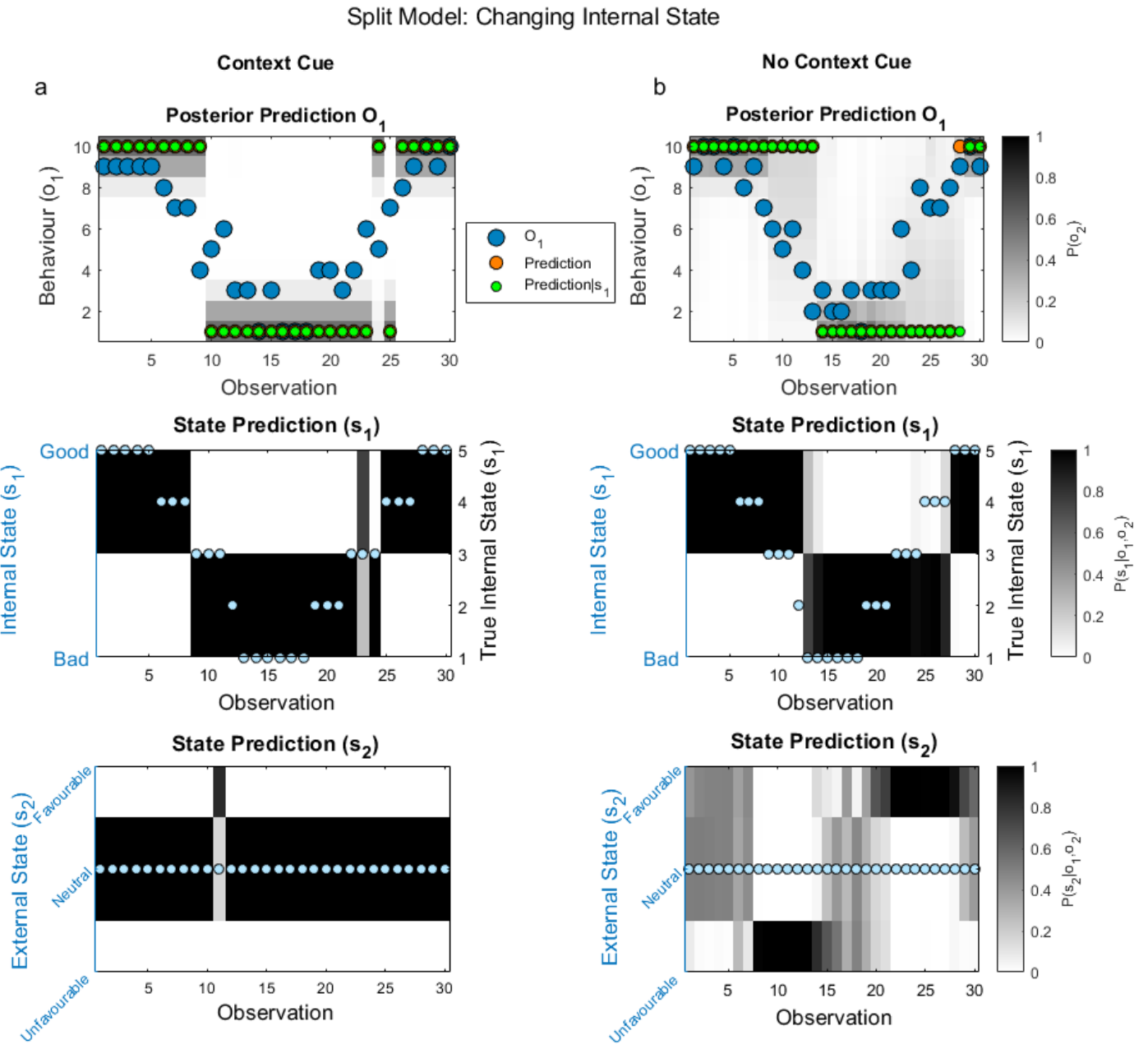

Note. Responses of a simulated subject with a frist-level split generative model, as shown in

Figure 3. Observed behaviour $\left(o_{1}\right)$ and cues $\left(o_{2}\right)$ are generated from a model in which internal state $\left(s_{1}\right)$ changes, while the external context $\left(s_{2}\right)$ remains constant. Notation and markers are as shown in Figure 6; note here that true internal states (light blue circles in the middle and lower rows) are generated from a graded model, with five levels, while the subject's model of internal state has only two levels. a) Given reliable information about external states, beliefs about internal state switch readily and symmetrically between inferring Good and Bad intent. b) In the absence of external state information, idealization and devaluation ensue. The subject initially persists in inferring Good intent, and attributes worsening behaviour to 
Unfavourable external factors (i.e. an excuse). The subject switches to inferring Bad intent only after seeing 'inexcusable' behaviour. After switching to infer Bad intent, the subject attributes improving behaviour to Favourable external factors (i.e. an ulterior motive). The subject switches back to inferring Good intentions only after seeing exemplary behaviour.

\section{Adjusting Attribution Errors: Excuses and Ulterior Motives}

A tendency towards idealization or devaluation can be adjusted by altering the scope of observations accounted for by external factors. Without an external state dimension, phases of idealization and devaluation are unstable; the subject switches beliefs readily from Good to Bad and vice versa (Figure 7a). Adding a Favourable external factor increases the range of behaviour consistent with Bad intent, and thereby produces a tendency to devalue others (Figure 7b). We suggest that in many cases 'Favourable external conditions' might be more fittingly conceptualized as an additional internal factor in the form of an ulterior motive. Thus, adding an ulterior motive instantiates a belief that people with Bad intentions may supply positive outcomes (e.g. to deceive) but people with Good intentions do not supply negative ones. Such a belief structure ensures that only the very best outcomes are interpreted as evidence of Good intent, while a wide range of outcomes are consistent with Bad intent. This accords with a description of paranoid personality disorder, as characterized by "misconstruing the neutral or friendly actions of others as hostile or contemptuous", and a "preoccupation with unsubstantiated 'conspiratorial' explanations of events"(World Health Organization, 1992). Conversely, adding only an Unfavourable external factor increases the range of 'excusable' behaviour and produces a tendency towards idealization (Figure 7c). 


\section{Figure 7}

Attribution Errors of a Split Model: Ulterior Motives and Excuses
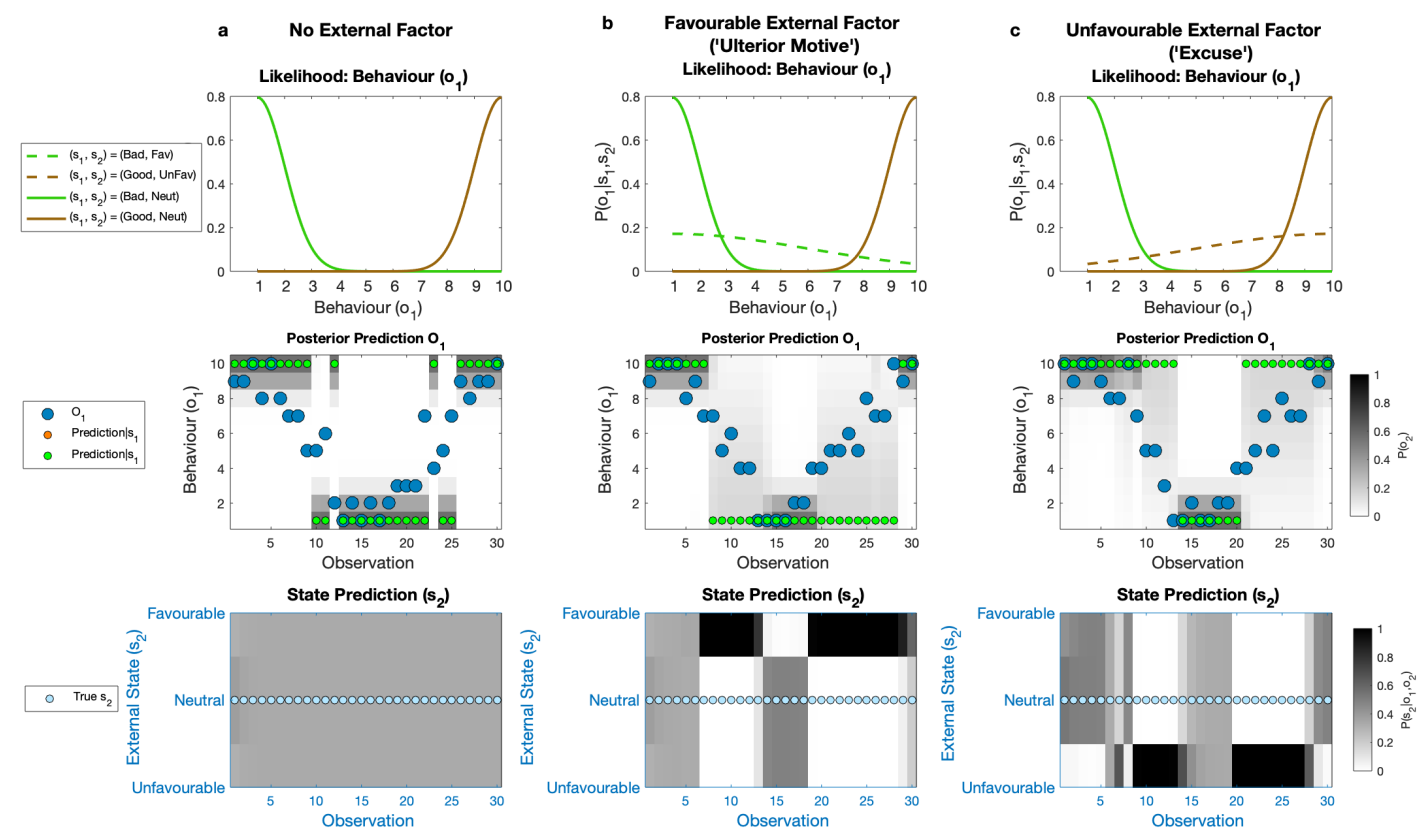

State Prediction $\left(\mathbf{s}_{2}\right)$
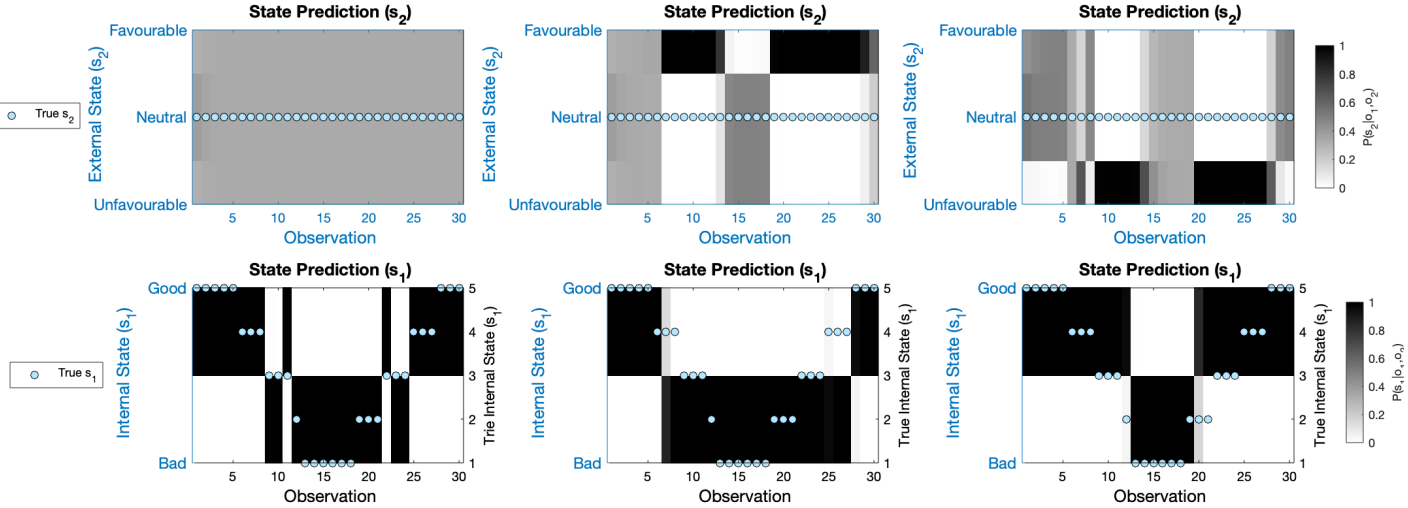

Note. Responses of a simulated subject with a split generative model. Notation and markers are as shown in Figure 5. a) Without an external state factor, beliefs about internal state switch readily and symmetrically between inferring Good and Bad intent. b) Adding an Favourable external factor ('ulterior motive'), increases the scope of behaviour consistent with Bad intentions, and produces a tendency to devalue others. c) Adding an Unfavourable external factor ('excuse'), increases the scope of behaviour consistent with Good intentions, and produces a tendency to idealise others.

\section{Retrospective Devaluation: Reappraisal of Past Observations}

The HMM's presented above include a propensity for others' internal states to change over time, encapsulated as random noise in the transition matrix. In other words, the subject's 
model includes a possibility that the other's intentions might change from Good to Bad or vice versa. By contrast, ruptures in cooperation seen in human relationships can be associated with a feeling that the other has 'shown their true colours', and were Bad all along, rather than that the other has changed, or that the other's Bad intent might be only transient. This pattern makes it difficult to leverage past experience to rebuild trust following ruptures. Such profound splitting results if the subject lacks a model of changes in intentional state. This can be implemented by setting noise in the transition matrix close to zero, such that the probability of internal state changes is very low. Good and Bad intent are then seen as enduring, mutually exclusive possibilities.

Under this model, when the subject switches from idealization to devaluation, previous observations are re-appraised. This is achieved in our model by the message-passing algorithm we use to invert the generative model, whereby beliefs regarding the causes of previous observations are updated after observing each new observation. Specifically, a single encounter consists of $T$ observations. After each observation, $t \leq T$, messages are passed 'forward' to update expectations about hidden states at each time point, $2 \leq \tau \leq T$, based on beliefs about the preceding time point, $\tau-1$. Similarly, 'backward messages' update expectations at each time point, $1 \leq \tau \leq T-1$, based on beliefs about the subsequent time point, $\tau+1$. (Note $\tau$ here refers to simulated time points within an encounter, distinct from its usage in Figure 1 to denote nested timescales). Thus, beliefs about current hidden states act as priors to constrain inference about past states, their relation to one-another being determined by the transition matrix. The resulting expectations regarding past hidden states at different time points during a trial are shown in Figure 8. Notably, after the subject switches from idealization to devaluation, previous good behaviour is reappraised as resulting from Bad intent with Favourable external circumstances (i.e. an ulterior motive). 


\section{Figure 8}

Retrospective Devaluation under a Split Model
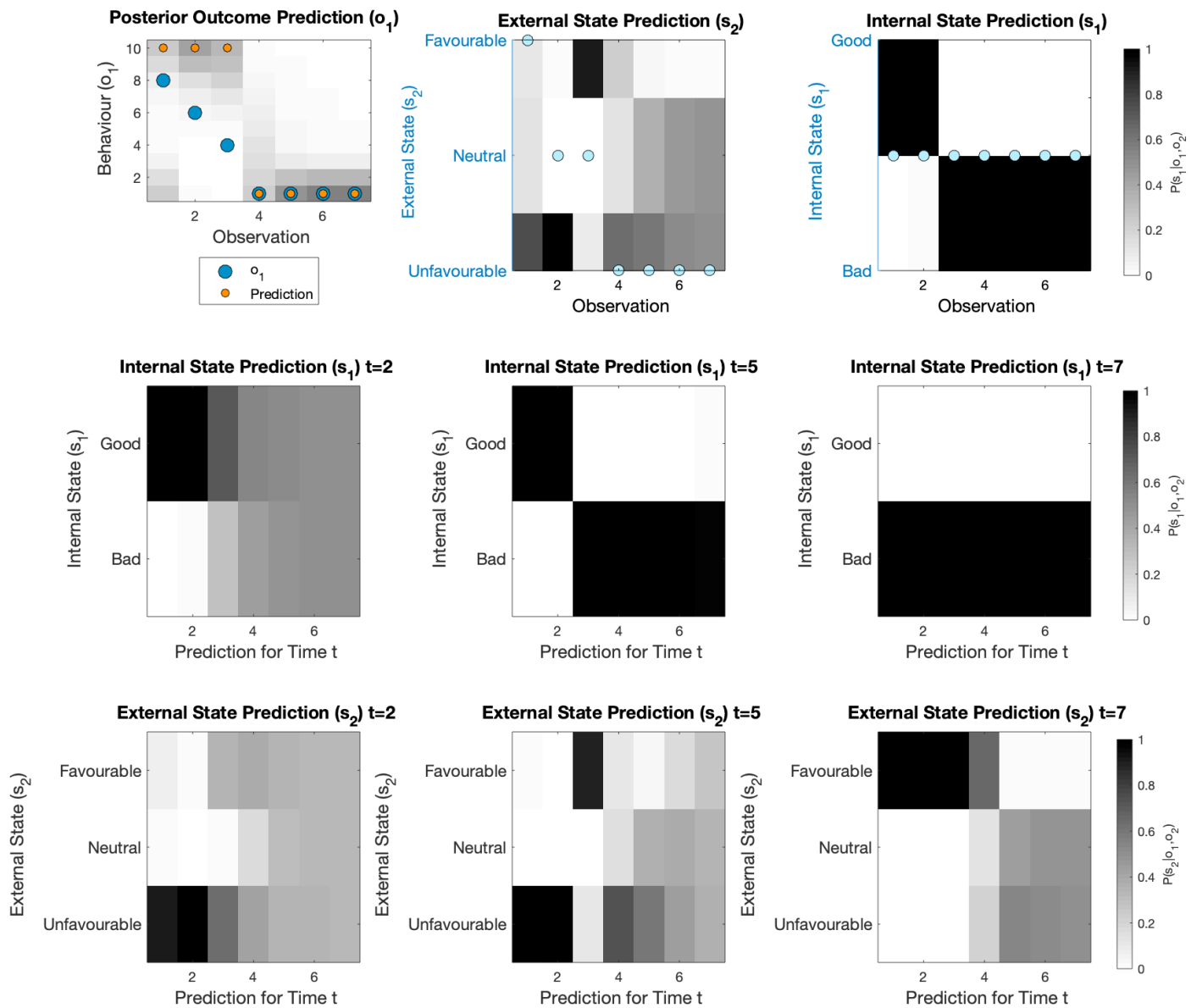

Note. Responses of a simulated subject with a split generative model with near-zero transition probabilities. Seven observations are shown, with notation as in Figure 5. The subject's posterior beliefs about outcomes and hidden states at each time point, $\tau=t$, are shown in the top row. The bottom two rows illustrate beliefs about hidden states at all previous and future time points, $1 \leq \tau \leq 7$, after the second ( $t=2)$, fifth $(t=5)$ and seventh $(t=7)$ observations. Following a switch to devaluation at $t=3$, previous instances of good behaviour are re-appraised and attributed to Bad intent with Favourable external conditions (i.e. an ulterior motive). This arises due to backward-message passing, whereby beliefs about current hidden states act as priors for inference about past states. 


\section{Higher-Order Splitting versus Integration}

Finally, we consider the possibility that splitting might arise further up an hierarchy of representation. To do so we examine a bimodal prior belief, $s_{3}$, over internal states. Here, the subject has a graded representation (healthy model) of internal states at the lower level, and is therefore capable of inferring a range of intentions. However, inference is shaped by a prior expectation that the other exists in two mutually exclusive 'modes', generating either Good or Bad intentions. We suggest that this model implies that a person has a capacity to differentiate grades of emotion, and is therefore capable of tracking subtle changes in others' emotional states, but remains prone to expect that other people will behave in polarized ways. We control the degree of splitting by symmetrically varying the separation between the two modes, termed $d$ ' . To emphasise effects of prior beliefs, we simulate only two observations per encounter. 


\section{Figure 9}

Hierarchical Splitting vs Integration

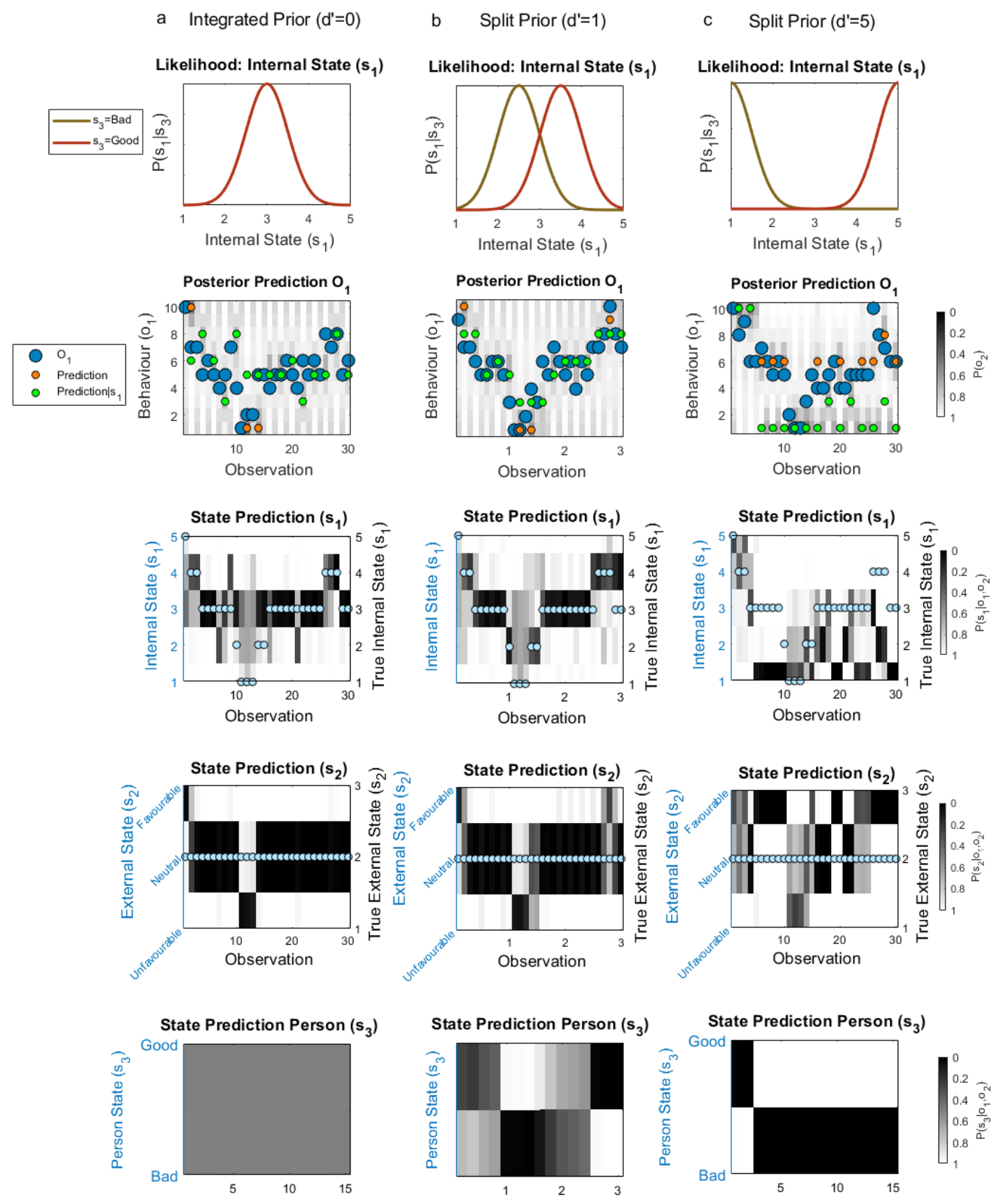

Note. Inference of a graded model of internal state, with the addition of a higher order state factor, $s_{3}$, acting as a prior, $P\left(s_{1} \mid s_{3}\right)$, over internal states at the level below, and corresponding to a model of another's character or personality. We show inference for 15 encounters, with two observations per encounter. Context cues are uninformative. The degree of splitting is controlled by symmetrically varying the separation between two modes of the 
prior, termed $d^{\prime}$. a) With a unimodal prior, inference about internal state is constrained, and more extreme behaviour is attributed to external factors. b) With a bimodal prior, the subject exhibits more extreme inference about internal state. c) With marked bimodality, idealization and devaluation result. For example, from the third observation onwards, the subject devalues the other, and assigns improving behaviour to Favourable external circumstances (i.e. an ulterior motive).

As shown in Figure 9a, when $d^{\prime}=0$, the subject has an integrated model of the other's intentions, which are expected to start as Neutral, with some uncertainty around this estimate. The subject's inference about the other's internal state is accordingly constrained by the prior. Since extreme internal states are less likely a priori, more extreme behaviour is instead attributed to external factors. This corresponds to a common-sense notion of behaviour that is 'out of character'. An integrated higher-order representation also loosely resembles a psychoanalytic notion of object constancy; the infant who has achieved object constancy can attribute changes in the care it receives to transient changes in the outside world (e.g., an absent mother) rather than to changes in internal states (e.g., a terrifying badness). With increasing $d^{\prime}$ (Figures 9a and 9b), inferred internal states become increasingly extreme, with explaining away at the first level further stabilising extreme beliefs. As expected, with increasing $d^{\prime}$, the subject predicts increasingly extreme behaviour, given the other's inferred internal state (Figure 10a). Importantly, with increasing $d^{\prime}$, the explanatory gain from adding an external state factor increases, predicting a correlation between extreme inference and paranoid attributions. In other words, people are more likely to include additional hidden factors to explain others' behaviour, to the extent that they hold split representations of others. Similarly, as splitting increases, subjects become more certain about internal state inferences (Figure 10b), and make increasing attribution errors (Figure 10c). 


\section{Figure 10}

Predictions associated with Splitting in a Hierarchical Model
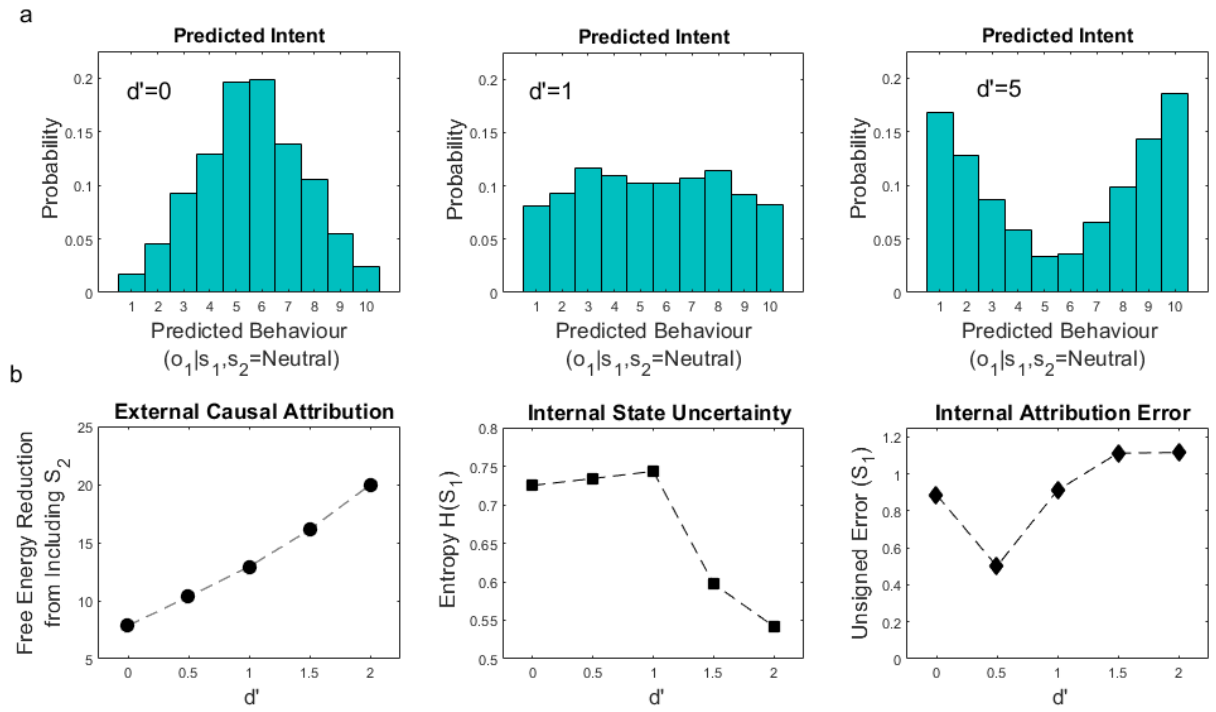

Note. a) Distribution of maximum a posteriori predictions about the others' behaviour, conditioned on internal state are shown for increasing degrees of splitting, after observing behaviour that changes according to a random walk. As expected, predictions about the other's 'intended behaviour' become increasingly polarized as d' increases. b) As splitting increases the model predicts a greater tendency to paranoid and conspiratorial explanations, greater certainty about internal states, and overall greater attribution error. Left panel: As d' increases, explanatory gain from adding an external state factor increases. Explanatory gain is measured by the improvement in model fit (reduction in variational free energy). Middle panel: Uncertainty over internal states, measured by Shannon entropy over posterior beliefs, decreases as splitting increases. Right panel: Attribution error, measured as the mean absolute difference between behaviour predicted from internal state and observed behaviour, is lowest at intermediate values of $d$ '. 


\section{Discussion}

In this paper we model 'splitting', or dichotomous thinking, from a Bayesian perspective. We formalise splitting as a polarized over-simplification in a person's model of others, such that behaviour arises from mutually exclusive internal states. This model leads to abrupt switches between idealization and devaluation, where each pole is stabilized by ‘explaining away' incongruent observations. During idealization, negative surprises are attributed to unfavourable external conditions, while during devaluation, positive surprises are attributed to favourable external conditions. Furthermore, we have shown how switches to devaluation are associated with retrospective appraisal that the other has 'shown their true colours', and were 'bad' all along, a pattern which makes it difficult to leverage past experience to rebuild trust. We contrast this state of affairs with a more nuanced generative model, consisting of a finer-grained representation, wherein inference is also constrained by longer timescale integration.

The model encompasses polarized appraisals of others that are often seen in several mental disorders. In particular, switches between idealization and devaluation are consistent with a pattern of "unstable and intense interpersonal relationships" seen in BPD (American Psychiatric Association, 2013). A combination of devaluation and external attributions for good behaviour also accords with features of paranoid personality disorder (World Health Organization, 1992), which is characterized by "misconstruing the neutral or friendly actions of others as hostile or contemptuous", and a "preoccupation with unsubstantiated 'conspiratorial' explanations of events". At the time of writing, there has been a recent move towards a dimensional, rather than categorical, classification of personality pathology (Bach \& First, 2018). Here, we have referred to specific personality disorders, but consider the proposed models naturally lend themselves to a dimensional approach. For example, a dimensional prediction of the model is that people who make more extreme evaluations of others' internal states also make more external causal attributions for changes in the other's behaviour. 


\section{Splits in Self-Representation}

As outlined in recent computational approaches (Smith, Kuplicki, et al., 2020; Smith, Lane, et al., 2019; Smith, Parr, et al., 2019), recognition of one's own emotions can be conceptualized as Bayesian inference. Thus, a formally identical scheme to that outlined in the simulations above can be applied to inference about the self. Here, observations might consist of feedback regarding one's own performance $\left(o_{1}\right)$ and external conditions $\left(o_{2}\right)$. For example, a tennis player might obtain feedback about the accuracy of their serve $\left(o_{1}\right)$, whilst also gauging any capricious gusts of wind that might deflect the ball $\left(\mathrm{O}_{2}\right)$. Internal hidden states would then entail an appraisal of performance or self-esteem.

With a split representation, patterns of idealization and devaluation of the self emerge. Instances of good feedback engender high self-esteem $\left(s_{2}=\operatorname{Good}\right)$; from this starting point, with poor performance tending to be explained away as due to external factors. This mechanism creates an inflated, grandiose sense of self, as observed chronically in narcissistic personalities and acutely in manic states. However, inflated self-appraisals would be liable to collapse in the face of particularly bad feedback. Devaluation of the self $\left(s_{2}=\mathrm{Bad}\right)$ would follow, at which point improvements in performance would be explained away, preventing recovery in self-esteem. Rapid collapses in self-esteem after poor performance resemble both narcissistic injury, and the self-punitive attributions seen in depression.

Self-inference can also be extended to higher-order representations of thoughts, feelings and actions across time. We have shown that a unimodal (integrated) higher-order prior constrains inference over internal states at the level below. Thus, an integrated prior ('ego') prevents extreme inference regarding one's own internal state. By contrast, with a split prior, internal state inference is inherently unstable. It appears plausible that disrupted self-inferential processes of this kind can lead to both the "markedly and persistently unstable 
self-image or sense of self" and "intense and unstable emotions" that characterise BPD

(American Psychiatric Association, 2013; Bender \& Skodol, 2007; Kernberg, 1967;

Kernberg, 1985; Koenigsberg, 2010).

\section{A Bayesian Explanation of Attribution Error}

Optimistic or self-serving biases are often explained by postulating that thoughts and beliefs hold value in and of themselves, such that people want to believe in their own good fortunes (Bromberg-Martin \& Sharot, 2020; Eil \& Rao, 2011; Hughes \& Zaki, 2015; Huys et al., 2012). Here no such additional values are required. When splitting is applied to the self, a self-serving bias results simply from a prior expectation that the self is Good, which ensures that evidence to the contrary is plausibly assigned to factors outside of the self. Similarly, a self-punitive bias results from a firm prior expectation that the self is Bad, such that evidence to the contrary is plausibly assigned to factors outside of the self. It is necessary to postulate neither a specific self-aggrandizing agency that becomes active when in an elevated state, nor a specific self-punitive agency that becomes active when self-esteem is low. Both biases result automatically from the mechanism of explaining away. Thus, the model presented here accounts for aspects of splitting that appear 'defensive' in a psychoanalytic sense, without postulating a specific defensive agency (see Zepf, 2012).

Spelled out explicitly, the reasoning creating attribution errors in our model is along the lines of either 'Given that I think I am [/the other is] exceptionally good, those apparent failings were the fault of something/somebody else', or 'Given that I think I am [/the other is] awful, those apparent successes were due to something/somebody else'. This arises out of the Bayesian nature of the model, wherein a subject arrives at the most plausible explanation for observations, given their (polarized) model of self or others. More balanced reasoning is rendered impossible by the restrictions of the person's split generative model. In other words, when a subject is faced with unexpected observations, rather than change their polarized view of self or others, the subject finds it worthwhile to adopt a more complex explanation, one that 
depends on poorly observed 'external' causes. Dorfman et al. (2019) illustrated this effect with a focus on the influence of external causes rather than internal splits. The authors show that people learn more from positive than from negative outcomes under conditions where external factors could worsen outcomes and learn more from negative than positive outcomes where external factors could improve outcomes. Gershman (2019) provided a similar perspective in terms of 'auxiliary hypotheses'.

The model we present accords with findings that people adjust causal attributions about their own performance in line with their expectations (Feather, 1969; Feather \& Simon, 1971a, 1971b). This makes an additional prediction that polarized beliefs about others are associated with a tendency towards excuse-making and conspiratorial explanations. Existing data provide indirect support for this idea. In an early study, (Streufert \& Streufert, 1969) examined attributions of success and failure made by pairs of participants playing a competitive decision-making game against a pre-programmed opponent, whom they believed to be another pair of participants. Participants were pre-grouped according to their conceptualizations of others: 'simple' subjects exhibited unidimensional interpersonal perceptions, while 'complex' subjects perceived others in a more multidimensional fashion. The authors predicted that simple subjects would be more prone to external attributions of failure than complex subjects, since complex subjects should be able to invoke additional internal dimensions with which to explain failure. As predicted, a self-serving effect was more marked in simple than in complex subjects: relative to complex subjects, simple subjects took more credit for success, and blamed failure on the opposing team to a greater extent. Our model furnishes these findings with a formal Bayesian interpretation, where 'simple' and 'complex' here are formalized as the granularity of an internal state representation.

\section{Dimensions of Splitting}


A key question for future investigation concerns the dimensions or decisions along which splitting occurs. Here, for simplicity, we have considered splitting along a single axis of valence, from Bad to Good. Interestingly, however, previous studies (Napolitano \& McKay, 2007; Sieswerda et al., 2005; Veen \& Arntz, 2000) have found that people with BPD sometimes make extreme emotional evaluations of opposite valence towards the same character, for instance rating someone as both highly reliable and highly jealous. Thus, in these studies, rather than classifying others as either 'entirely good' or 'entirely bad', BPD patients displayed dichotomous thinking along a range of dimensions. Veen and Arntz (2000) concluded that rather than showing unidimensional dichotomous thinking, as implied by classical psychoanalytic accounts of splitting, BPD patients display multidimensional dichotomous thinking.

Veen and Arntz (2000) noted that the design of their study prompted BPD participants to rate various pre-specified emotional dimensions, and might therefore have tended to promote multidimensional emotional thinking. Indeed, a follow-up study with the same participant groups (Arntz \& Veen, 2001) using an open-ended response format, found that both BPD and Cluster $\mathrm{C}$ personality disorder groups described the film characters as more affectively polarized, along fewer affective dimensions, when compared with controls, a pattern more consistent with classical accounts of splitting. Nevertheless, these findings draw attention the likelihood that splitting is not restricted to a single Bad-Good axis. An interesting direction for future work will be to extend the framework introduced here to encompass multiple emotional dimensions.

A further interesting consideration is whether splitting represents a pervasive reasoning style applying to various forms judgement, or alternatively is specific to social or emotional decisions. Existing studies suggest that dichotomous thinking in BPD is prominent only in relational situations pertaining to themes of abandonment, abuse or neglect (Sieswerda et al., 2005; Veen \& Arntz, 2000). In keeping with this idea, psychoanalytic theories propose that splitting comes to the fore under conditions of relational stress (Steiner, 1987, 1992). Similarly, mentalizing ability is thought to deteriorate at times of attachment 
stress (Agrawal et al., 2004; Bateman \& Fonagy, 2013; Choi-Kain et al., 2009; Fonagy \& Bateman, 2008; Lyons-Ruth et al., 2005; Nolte et al., 2013; Nolte et al., 2019). These ideas suggest that splits in BPD might pertain to particular forms of attachment relationship or relational schema; for example, 'badness' might specifically correspond to feelings of abandonment, or 'goodness' to an expectation of an all-fulfilling caregiver.

\section{Self-Other Interactions}

Here we make a simplifying assumption that a subject infers another's mental state from that person's behaviour. In reality, there is likely to be a complex interplay between inference about self and other. Indeed, several existing theories link relational instability to an impaired capacity to distinguish self and other (Bender et al., 2011; Bender \& Skodol, 2007; Kohut, 2013; Fonagy \& Luyten, 2009). Mentalization-based theory, for example describes how a person who perceives self and other as fused is directly vulnerable to the vicissitudes of another's reactions (Fonagy \& Target, 1996; Nolte et al., 2019), and may have a mistaken perception that their own mental states are directly accessible to others (Fonagy \& Luyten, 2009, p1363-1364). Extending the ideas presented here to encompass simultaneous inference about self and other is a pressing subject for future investigation.

\section{Existing Studies of Social Interaction in BPD}

The model presented here might illuminate existing experimental findings regarding social interaction and social inference in BPD. In an influential study, BPD players were less likely to recover cooperation following ruptures when playing an iterated trustee game (KingCasas et al., 2008), which resulted from a lower propensity of BPD trustees to 'coax' healthy investors to restore cooperation by maintaining higher offers following low investments. Hula et al. (2018) modelled these findings in terms of 'mentalizing' ability, formalized as the depth of a recursive representation of the other player's mental model. The authors postulate a state 
of 'irritability' following low offers, in which both mentalizing and empathy evaporate, at least temporarily. A trustee's ability to maintain higher offers then results from their having a model that the investor is irritated, motivating the trustee to act more generously to avoid increases in irritation. By contrast, BPD patients behaved as if they had lower awareness of other's irritability, giving rise to lower coaxing. Our model suggests an alternative possibility that lower offers might be misconstrued as inference that the investor is a 'bad person', thereby rendering further attempts at restoring cooperation futile. By contrast a graded model would allow the player to infer that lower offers from the investor could result from local changes in the investor's internal state.

Also pertinent to the current work is a recent study in which participants rated the moral character of two agents, after observing how much each agent was willing to trade-off money against painful shocks for a third person (Siegel et al., 2018). A 'Bad' agent was more inclined to take money at the expense of shocks for the other person, while a 'Good' agent was more charitable. Healthy participants were found to be faster to learn about Bad agents than Good, and were also more uncertain in their appraisals of the moral character of Bad agents. The authors suggest that observing bad behaviour primes feelings of threat, causing beliefs about others to become more uncertain and therefore more amenable to rapid updating. Such flexibility is said to promote vigilance against worsening behaviour, whilst also allowing beliefs to be amended if behaviour improves, thereby supporting recovery of cooperation.

In a further study using a similar design (Siegel et al., 2020), this asymmetry in updating was reduced in participants with $\mathrm{BPD}$, who held more certain, rigid beliefs about bad others compared with healthy controls. Consistent with previous findings, participants with BPD started with more negative appraisals of others. BPD participants were subsequently slower to learn about bad others and faster to learn about good others, relative to controls. Here, the authors suggest that faster learning about good others occurred because the good agent violated the (pessimistic) expectations of BPD participants to a greater extent than the bad agent. 
In our model, belief updating depends on the precision of the likelihood representations and volatility in the transition matrix. Asymmetries in updating as described by Siegel et al. (2020) might be therefore accounted for under our model by tuning transition beliefs to increase volatility in response to unexpectedly bad behaviour. More straightforwardly, faster updating for bad others could be explained by asymmetric higherorder splitting, specified such that a prior $\left(s_{3}\right)$ is less precise for 'Bad' others than for 'Good' others. Faster updating would then result from less precise priors, rather than changes in volatility. Psychologically this would entail a healthy intuition that people who behave badly also behave less predictably, and sometimes have good qualities. By contrast the pattern of results seen in BPD would imply a more precise 'Bad' prior, and a less precise 'Good' prior, relative to controls. This would imply an intuition, perhaps shaped by experiences of inconsistent or abusive parenting, that some people are predictably bad. Further investigation is needed to test these possibilities empirically.

\section{Social Learning}

In the present framework we have considered inference rather than learning. In the current context, learning entails updating the parameters of a generative model. A pertinent question with respect to learning is which developmental conditions promote acquisition of integrated or split representations. Fonagy and colleagues describe how a responsive parent takes-in and re-processes the child's emotional needs, communicating these back to the child in a digestible form. Through this process of 'contingent mirroring' the parent helps the child to identify its own emotional states and their causes (Fonagy, 2002; Gergely \& Watson, 1996; Target \& Fonagy, 1996). Similarly, psychoanalytic theorists have proposed that to fully achieve object constancy, the child needs the help of its caregivers to buffer extreme affective shifts (e.g. Fonagy, 2002; Gergely \& Watson, 1996; Target \& Fonagy, 1996; Mahler et al., 1975; for a review see Akhtar, 1994). Future, work might usefully focus on how such processes foster learning of a more integrated state representation. Finally, we note that, once 
established, split representations are likely to be resistant to learning on account of their prior precision, and further stabilized by the explaining away of countervailing observations.

\section{Summary}

We suggest that accurate inferences about others and the self are favoured by a finegrained representation of internal states, combined with an integrated representation of how internal states change across time. We have shown how unstable inference can result from various lesions in a generative model - namely, a polarized representation of how internal states generate behaviour, no representation of change in internal states across time, and/or an imprecise model of external context. Our model makes clear predictions that accord with established human relational phenomena, and invites further work to determine how these different lesions might be distinguished experimentally. 


\section{Acknowledgements}

The authors would like to thank Peter Fonagy for helpful discussion regarding this work.

\section{References}

Abramson, L. Y., Seligman, M. E., \& Teasdale, J. D. (1978). Learned helplessness in humans: critique and reformulation. Journal of Abnormal Psychology, 87(1),

49. https://doi.org/10.1037/0021-843X.87.1.49

Agrawal, H. R., Gunderson, J., Holmes, B. M., \& Lyons-Ruth, K. (2004). Attachment studies with borderline patients: A review. Harvard Review of Psychiatry, 12(2), 94-104. https://doi.org/10.1080/10673220490447218

Akhtar, S. (1994). Object constancy and adult psychopathology. International Journal of Psycho-analysis, 75, 441-455. PMID: 7960426

Akhtar, S., \& Byrne, J. P. (1983). The concept of splitting and its clinical relevance. The American Journal of Psychiatry, 140(8), 10131016. https://doi.org/10.1176/ajp.140.8.1013

American Psychiatric Association (2013). Diagnostic and statistical manual of mental disorders, (DSM-5®). Author. https://doi.org/10.1176/appi.books.9780890425596.dsm05

Arntz, A., Dietzel, R., \& Dreessen, L. (1999). Assumptions in borderline personality disorder: Specificity, stability and relationship with etiological factors. Behaviour Research and Therapy, 37(6), 545-557. https://doi.org/10.1016/S00057967(98)00152-1

Arntz, A., \& Veen, G. (2001). Evaluations of others by borderline patients. The Journal of Nervous and Mental disease, 189(8), 513-521. https://doi.org/doi: 10.1097/00005053200108000-00004 
Bach, B., First, M.B. (2018). Application of the ICD-11 classification of personality disorders. BMC Psychiatry 18, 351. https://doi.org/10.1186/s12888-018-1908-3

Barnby, J. M., Deeley, Q., Robinson, O., Raihani, N., Bell, V., \& Mehta, M. A. (2020). Paranoia, sensitization and social inference: findings from two large-scale, multiround behavioural experiments. Royal Society Open Science, 7(3), 191525. https://doi.org/10.1098/rsos.191525

Barrett, L. F. (2006). Are emotions natural kinds? Perspectives on Psychological Science, l(1), 28-58. https://doi.org/10.1111/j.1745-6916.2006.00003.x

Bateman, A., \& Fonagy, P. (2013). Mentalization-based treatment. Psychoanalytic Inquiry, 33(6), 595-613. https://doi.org/10.1080/07351690.2013.835170

Bateman, A. W., Gunderson, J., \& Mulder, R. (2015). Treatment of personality disorder. The Lancet, 385(9969), 735-743. https://doi.org/10.1016/S0140-6736(14)61394-5

Beck, A. T. (1963). Thinking and depression: I. Idiosyncratic content and cognitive distortions. Archives of General Psychiatry, 9(4), 324-333. https://doi.org/doi:10.1001/archpsyc.1963.01720160014002

Beck, A. T., Butler, A. C., Brown, G. K., Dahlsgaard, K. K., Newman, C. F., \& Beck, J. S. (2001). Dysfunctional beliefs discriminate personality disorders. Behaviour Research and Therapy, 39(10), 1213-1225. https://doi.org/10.1016/S0005-7967(00)00099-1

Beck, A. T., Davis, D. D., \& Freeman, A. (Eds.). (2015). Cognitive therapy of personality disorders. Guilford Publications.

Bender, D. S., Morey, L. C., \& Skodol, A. E. (2011). Toward a model for assessing level of personality functioning in DSM-5, part I: A review of theory and methods. Journal of Personality Assessment, 93(4), 332-346. https://doi.org/10.1080/00223891.2011.583808

Bender, D. S., \& Skodol, A. E. (2007). Borderline personality as a self-other representational disturbance. Journal of Personality Disorders, 21(5), 500-517. https://doi.org/10.1521/pedi.2007.21.5.500 
Bhar, S. S., Brown, G. K., \& Beck, A. T. (2008). Dysfunctional beliefs and psychopathology in borderline personality disorder. Journal of Personality Disorders, 22(2), 165-177. https://doi.org/10.1521/pedi.2008.22.2.165

Bishop, C. M. (2006). Sequential Data. In C. M. Bishop, Pattern recognition and machine learning (pp. 605-652). Springer.

Block, J. (1982). Assimilation, accommodation, and the dynamics of personality development. Child Development, 281-295. https://doi.org/10.2307/1128971

Bradley, G. W. (1978). Self-serving biases in the attribution process: A reexamination of the fact or fiction question. Journal of Personality and Social Psychology, 36(1),

56. https://doi.org/10.1037/0022-3514.36.1.56

Bromberg-Martin, E. S., \& Sharot, T. (2020). The value of beliefs. Neuron, 106(4), 561-565. https://doi.org/10.1016/j.neuron.2020.05.001

Butler, A. C., Brown, G. K., Beck, A. T., \& Grisham, J. R. (2002). Assessment of dysfunctional beliefs in borderline personality disorder. Behaviour Research and Therapy, 40(10), 1231-1240. https://doi.org/10.1016/S0005-7967(02)00031-1

Chater, N., \& Oaksford, M. (Eds.). (2008). The probabilistic mind: Prospects for Bayesian cognitive science. Oxford University Press.

Choi-Kain, L. W., Fitzmaurice, G. M., Zanarini, M. C., Laverdière, O., \& Gunderson, J. G. (2009). The relationship between self-reported attachment styles, interpersonal dysfunction, and borderline personality disorder. The Journal of Nervous and Mental Disease, 197(11), 816-821. https://doi.org/10.1097/NMD.0b013e3181bea56e

Del Pozo, M. A., Harbeck, S., Zahn, S., Kliem, S., \& Kröger, C. (2018). Cognitive distortions in anorexia nervosa and borderline personality disorder. Psychiatry Research, 260, 164-172. https://doi.org/10.1016/j.psychres.2017.11.043

Diaconescu, A. O., Wellstein, K. V., Kasper, L., Mathys, C., \& Stephan, K. E. (2020). Hierarchical Bayesian models of social inference for probing persecutory delusional ideation. Journal of Abnormal Psychology, 129(6), 556.

https://doi.org/10.1037/abn0000500 
Dorfman, H. M., Bhui, R., Hughes, B. L., \& Gershman, S. J. (2019). Causal inference about good and bad outcomes. Psychological Science, 30(4), 516-525. https://doi.org/10.1177/0956797619828724

Dunsmoor, J. E., Niv, Y., Daw, N., \& Phelps, E. A. (2015). Rethinking extinction. Neuron, 88(1), 47-63. https://doi.org/10.1016/j.neuron.2015.09.028

Eil, D., \& Rao, J. M. (2011). The good news-bad news effect: asymmetric processing of objective information about yourself. American Economic Journal: Microeconomics, 3(2), 114-138. https://doi.org/ 10.1257/mic.3.2.114

Fairbairn, W. R. D. (1940). Schizoid factors in the personality. In W. R. D. Fairbairn Psychoanalytic studies of the personality. Routledge. (1952) (Reprinted from 1992)

Fairbairn, W. R. D. (1952). Psychoanalytic studies of the personality. Tavistock Publications. Feather, N. T. (1969). Attribution of responsibility and valence of success and failure in relation to initial confidence and task performance. Journal of Personality and Social Psychology, 13(2), 129. https://doi.org/10.1037/h0028071

Feather, N. T., \& Simon, J. G. (1971a). Attribution of responsibility and valence of outcome in relation to initial confidence and success and failure of self and other. Journal of Personality and Social Psychology, 18(2), 173. https://doi.org/10.1037/h0030845

Feather, N. T., \& Simon, J. G. (1971b). Causal attributions for success and failure in relation to expectations of success based upon selective or manipulative control. Journal of Personality, 39(4), 527-541. https://doi.org/10.1111/j.1467-6494.1971.tb00060.x

Feldman, M. (1992). Splitting and projective identification. In R. Anderson (Ed.). Clinical lectures on Klein and Bion (pp. 74-88). Tavistock/Routledge.

Fitch, G. (1970). Effects of self-esteem, perceived performance, and choice on causal attributions. Journal of Personality and Social Psychology, 16(2), 311. https://doi.org/10.1037/h0029847

Fonagy, P. (2002). The internal working model or the interpersonal interpretive function. Journal of Infant, Child, and Adolescent Psychotherapy, 2(4), 27-38. https://doi.org/10.1080/15289168.2002.10486417 
Fonagy, P., \& Bateman, A. (2008). The development of borderline personality disorder-A mentalizing model. Journal of Personality Disorders, 22(1), 4-21. https://doi.org/10.1521/pedi.2008.22.1.4

Fonagy, P., \& Luyten, P. (2009). A developmental, mentalization-based approach to the understanding and treatment of borderline personality disorder. Developmental Psychopathology, 21(4), 1355-1381. https://doi.org/10.1017/S0954579409990198

Fonagy, P., \& Target, M. (1996). Playing with reality: I. Theory of mind and the normal development of psychic reality. International Journal of Psycho-analysis, 77, $217-$ 233. PMID: 8771375

Forgas, J. P., Bower, G. H., \& Moylan, S. J. (1990). Praise or blame? Affective influences on attributions for achievement. Journal of Personality and Social Psychology, 59(4), 809. https://doi.org/10.1037/0022-3514.59.4.809

Freud, A. (1965). Normality and pathology in childhood: Assessments of development. Routledge.

Friston, K., FitzGerald, T., Rigoli, F., Schwartenbeck, P., \& Pezzulo, G. (2016). Active inference and learning. Neuroscience \& Biobehavioral Reviews, 68, 862-879. https://doi.org/10.1016/j.neubiorev.2016.06.022

Friston, K., FitzGerald, T., Rigoli, F., Schwartenbeck, P., \& Pezzulo, G. (2017). Active inference: a process theory. Neural computation, 29(1), 1-49. https://doi.org/10.1162/NECO_a 00912

Geiger, P. J., Peters, J. R., \& Baer, R. A. (2014). Using a measure of cognitive distortion to examine the relationship between thought suppression and borderline personality features: A multi-method investigation. Personality and Individual Differences, 59, 54-59. https://doi.org/10.1016/j.paid.2013.11.005

Gergely, G., \& Watson, J. S. (1996). The social biofeedback theory of parental affectmirroring: the development of emotional self-awareness and self-control. International Journal of Psycho-analysis, 77, 1181-1212. PMID: 9119582 
Gershman, S. J., \& Niv, Y. (2012). Exploring a latent cause theory of classical conditioning. Learning \& Behavior, 40(3), 255-268. https://doi.org/10.3758/s13420012-0080-8

Gershman, S. J. (2017). Context-dependent learning and causal structure. Psychonomic Bulletin \& Review, 24(2), 557-565. https://doi.org/10.3758/s13423-016-1110-x

Gershman, S. J. (2019). How to never be wrong. Psychonomic Bulletin \& Review, 26(1), 1328. https://doi.org/10.3758/s13423-018-1488-8

Gershman, S. J., \& Blei, D. M. (2012). A tutorial on Bayesian nonparametric models. Journal of Mathematical Psychology, 56(1), 1-12. https://doi.org/10.1016/j.jmp.2011.08.004

Gershman, S. J., Jones, C. E., Norman, K. A., Monfils, M.-H., \& Niv, Y. (2013). Gradual extinction prevents the return of fear: implications for the discovery of state. Frontiers in Behavioural Neuroscience, 7, 164. https://doi.org/10.3389/fnbeh.2013.00164

Gershman, S. J., Norman, K. A., \& Niv, Y. (2015). Discovering latent causes in reinforcement learning. Current Opinion in Behavioral Sciences, 5, 43-50. https://doi.org/10.1016/j.cobeha.2015.07.007

Glimcher, P. W. (2004). Decisions, uncertainty, and the brain: The science of neuroeconomics. MIT press.

Gomez, L. (1997). An introduction to object relations. NYU Press.

Griffiths, T., \& Yuille, A. (2008). A primer on probabilistic inference. In N. Chater, \& M. Oaksford (Eds.). The probabilistic mind: Prospects for Bayesian cognitive science (pp. 33-57). Oxford University Press.

Harter, S., \& Buddin, B. J. (1987). Children's understanding of the simultaneity of two emotions: A five-stage developmental acquisition sequence. Developmental Psychology, 23(3), 388. https://doi.org/10.1037/0012-1649.23.3.388

Harter, S., \& Whitesell, N. R. (1989). Developmental changes in children's understanding of single, multiple, and blended emotion concepts. In C. Saarni \& P. L. Harris 
(Eds.), Children's understanding of emotion (pp. 81-116). Cambridge University Press.

Hartmann, H. (1952). The mutual influences in the development of ego and id. The Psychoanalytic Study of the Child, 7(1), 9-30. https://doi.org/10.1080/00797308.1952.11823149

Hinshelwood, R. D. (1989). A dictionary of Kleinian thought. Free Association Books.

Hoemann, K., Wu, R., LoBue, V., Oakes, L. M., Xu, F., \& Barrett, L. F. (2020). Developing an understanding of emotion categories: Lessons from objects. Trends in Cognitive Sciences, 24(1), 39-51. https://doi.org/10.1016/j.tics.2019.10.010

Horowitz, L. M. (2004). Interpersonal foundations of psychopathology. American Psychological Association.

Hughes, B. L., \& Zaki, J. (2015). The neuroscience of motivated cognition. Trends in Cognitive Sciences, 19(2), 62-64. https://doi.org/10.1016/j.tics.2014.12.006

Hula, A., Vilares, I., Lohrenz, T., Dayan, P., \& Montague, P. R. (2018). A model of risk and mental state shifts during social interaction. PLoS Computational Biology, 14(2), e1005935. https://doi.org/10.1371/journal.pcbi.1005935

Huys, Q. J., Eshel, N., O'Nions, E., Sheridan, L., Dayan, P., \& Roiser, J. P. (2012). Bonsai trees in your head: how the Pavlovian system sculpts goal-directed choices by pruning decision trees. PLoS Computational Biology, 8(3), e1002410. https://doi.org/10.1371/journal.pcbi.1002410

Kernberg, O. (1967). Borderline personality organization. Journal of the American Psychoanalytic Association, 15(3), 641-685. https://doi.org/10.1177/000306516701500309

Kernberg, O. F. (1985). Borderline conditions and pathological narcissism. Rowman \& Littlefield.

Kernberg, O. F. (2015). Neurobiological correlates of object relations theory: The relationship between neurobiological and psychodynamic development. International Forum of Psychoanalysis, 24(1), 38-46. https://doi.org/10.1080/0803706X.2014.912352 
King-Casas, B., Sharp, C., Lomax-Bream, L., Lohrenz, T., Fonagy, P., \& Montague, P. R. (2008). The rupture and repair of cooperation in borderline personality disorder. Science, 321(5890), 806-810. https://doi.org/10.1126/science.1156902

Klein, M. (1946). Notes on some schizoid mechanisms. International Journal of Psychoanalysis, 27, 99-110. PMID: 22700275

Koenigsberg, H. W. (2010). Affective instability: toward an integration of neuroscience and psychological perspectives. Journal of Personality Disorders, 24(1), 60-82. https://doi.org/10.1521/pedi.2010.24.1.60

Kohut, H. (1966). Forms and transformations of narcissism. Journal of the American Psychoanalytic Association, 14(2), 243-272. https://doi.org/10.1177/000306516601400201

Kohut, H. (2013). The analysis of the self: A systematic approach to the psychoanalytic treatment of narcissistic personality disorders. University of Chicago Press.

Kramer, U., de Roten, Y., Perry, J. C., \& Despland, J.-N. (2013). Beyond splitting: Observerrated defense mechanisms in borderline personality disorder. Psychoanalytic Psychology, 30(1), 3. https://doi.org/10.1037/a0029463

Lyons-Ruth, K., Yellin, C., Melnick, S., \& Atwood, G. (2005). Expanding the concept of unresolved mental states: Hostile/helpless states of mind on the Adult Attachment Interview are associated with disrupted mother-infant communication and infant disorganization. Development and Psychopathology, 17(1), 1. PMID: 15971757

Mahler, M. S., Pine, F., \& Bergman, A. (1975). The psychological birth of the human infant. symbiosis and individuation. Basic Books. https://doi.org/10.4324/9780429482915

Maruna, S., \& Mann, R. E. (2006). A fundamental attribution error? Rethinking cognitive distortions. Legal and Criminological Psychology, 11(2), 155-177. https://doi.org/10.1348/135532506X114608

McCormack, T., Frosch, C., Patrick, F., \& Lagnado, D. (2015). Temporal and statistical information in causal structure learning. Journal of Experimental Psychology: Learning, Memory, and Cognition, 41(2), 395. https://doi.org/10.1037/a0038385 
Miller, D. T., \& Ross, M. (1975). Self-serving biases in the attribution of causality: Fact or fiction? Psychological Bulletin, 82(2), 213. https://doi.org/10.1037/h0076486

Moritz, S., Schilling, L., Wingenfeld, K., Köther, U., Wittekind, C., Terfehr, K., \& Spitzer, C. (2011). Psychotic-like cognitive biases in borderline personality disorder. Journal of Behavior Therapy and Experimental Psychiatry, 42(3), 349-354. https://doi.org/10.1016/j.jbtep.2011.02.003

Moutoussis, M., Fearon, P., El-Deredy, W., Dolan, R. J., \& Friston, K. J. (2014). Bayesian inferences about the self (and others): A review. Consciousness and Cognition, 25, 67-76. https://doi.org/10.1016/j.concog.2014.01.009

Moutoussis, M., Trujillo-Barreto, N. J., El-Deredy, W., Dolan, R., \& Friston, K. (2014). A formal model of interpersonal inference. Frontiers in Human Neuroscience, 8, 160. https://doi.org/10.3389/fnhum.2014.00160

Moutoussis, M., El-Deredy, W., \& Bentall, R. P. (2015). An empirical study of defensive avoidance in Paranoia. Behavioural and Cognitive Psychotherapy, 43(2), 182. https://doi.org/ 10.1017/S1352465813000805

Napolitano, L. A., \& McKay, D. (2007). Dichotomous thinking in borderline personality disorder. Cognitive Therapy and Research, 31(6), 717-726. https://doi.org/ $\underline{10.1007 / \mathrm{s} 10608-007-9123-4}$

Nisbett, R. E., \& Ross, L. (1980). Human inference: Strategies and shortcomings of social judgment. Prentice Hall.

Nolte, T., Bolling, D. Z., Hudac, C., Fonagy, P., Mayes, L. C., \& Pelphrey, K. A. (2013). Brain mechanisms underlying the impact of attachment-related stress on social cognition. Frontiers in Human Neuroscience, 7 , 816. https://doi.org/10.3389/fnhum.2013.00816

Nolte, T., Campbell, C., \& Fonagy, P. (2019). A mentalization-based and neuroscienceinformed model of severe and persistent psychopathology. In J. G. Pereira, J. Gonçalves, V. Bizzari (Eds.). The neurobiologypsychotherapy-pharmacology 
intervention triangle: The need for common sense in 21st century mental health (pp. 161-184). Vernon Press.

Noorani, I., \& Carpenter, R. H. S. (2016). The LATER model of reaction time and decision. Neuroscience \& Biobehavioral Reviews, 64, 229-251. https://doi.org/10.1016/j.neubiorev.2016.02.018

Parr, T., Markovic, D., Kiebel, S. J., \& Friston, K. J. (2019). Neuronal message passing using Mean-field, Bethe, and Marginal approximations. Scientific reports, 9(1), 1-18. https://doi.org/10.1038/s41598-018-38246-3

Perry, J. C., Presniak, M. D., \& Olson, T. R. (2013). Defense mechanisms in schizotypal, borderline, antisocial, and narcissistic personality disorders. Psychiatry: Interpersonal \& Biological Processes, 76(1), 32-52. https://doi.org/10.1521/psyc.2013.76.1.32

Piaget, J. (2013). The construction of reality in the child. Routledge.

Piaget, J. (1954). The development of causality (M. Cook, Trans.). In J. Piaget \& M. Cook (Trans.), The construction of reality in the child (pp. 219-319). Basic Books. https://doi.org/10.1037/11168-003

Preißler, S., Dziobek, I., Ritter, K., Heekeren, H. R., \& Roepke, S. (2010). Social cognition in borderline personality disorder: evidence for disturbed recognition of the emotions, thoughts, and intentions of others. Frontiers in Behavioural Neuroscience, 4, 182. https://doi.org/10.3389/fnbeh.2010.00182

Pretzer, J. L., \& Beck, A. T. (1996). A cognitive theory of personality disorders. IN M. F. Lenzenweger \& J. F. Clarkin (Eds.). Major theories of personality disorder (pp. 36105). Guildford.

Reiter, A. M. F., Diaconescu, A. O., Eppinger, B., \& Li, S.-C. (2019). Human aging alters Bayesian social inference about others' changing intentions. https://psyarxiv.com/3etup/download?format=pdf 
Roepke, S., Vater, A., Preißler, S., Heekeren, H. R., \& Dziobek, I. (2013). Social cognition in borderline personality disorder. Frontiers in Neuroscience, 6, 195. https://doi.org/10.3389/fnins.2012.00195

Ross, L. (1977). The intuitive psychologist and his shortcomings: Distortions in the attribution process. Advances in Experimental Social Psychology, 10, 173-220. https://doi.org/10.1016/S0065-2601(08)60357-3

Seligman, M. E., Abramson, L. Y., Semmel, A., \& Von Baeyer, C. (1979). Depressive attributional style. Journal of Abnormal Psychology, 88(3), 242. https://doi.org/10.1037/0021-843X.88.3.242

Seligman, M. E., \& Schulman, P. (1986). Explanatory style as a predictor of productivity and quitting among life insurance sales agents. Journal of Personality and Social Psychology, 50(4), 832. https://doi.org/10.1037/0022-3514.50.4.832

Seligman, M. E. P. (1991). Learned optimism: How to change your mind and your life. Knopf.

Siegel, J. Z., Curwell-Parry, O., Pearce, S., Saunders, K. E. A., \& Crockett, M. J. (2020). A computational phenotype of disrupted moral inference in borderline personality disorder. Biological Psychiatry: Cognitive Neuroscience and Neuroimaging, 5(12), 1134-1141. https://doi.org/10.1016/j.bpsc.2020.07.013

Siegel, J. Z., Mathys, C., Rutledge, R. B., \& Crockett, M. J. (2018). Beliefs about bad people are volatile. Nature Human Behaviour, 2(10), 750-756. https://doi.org/10.1038/s41562-018-0425-1

Sieswerda, S., Arntz, A., \& Wolfis, M. (2005). Evaluations of emotional noninterpersonal situations by patients with borderline personality disorder. Journal of Behavior therapy and Experimental Psychiatry, 36(3), 209-225. https://doi.org/10.1016/j.jbtep.2005.05.004

Smith, R., Friston, K., \& Whyte, C. (2021). A step-by-step tutorial on active inference and its application to empirical data. https://psyarxiv.com/b4jm6/. 
Smith, R., Killgore, W. D. S., \& Lane, R. D. (2018). The structure of emotional experience and its relation to trait emotional awareness: A theoretical review. Emotion, 18(5), 670. https://doi.org/10.1037/emo0000376

Smith, R., Kuplicki, R., Feinstein, J., Forthman, K. L., Stewart, J. L., Paulus, M. P., Tulsa, i., \& Khalsa, S. S. (2020). A Bayesian computational model reveals a failure to adapt interoceptive precision estimates across depression, anxiety, eating, and substance use disorders. PLoS Computational Biology, 16(12), e1008484. https://doi.org/10.1371/journal.pcbi.1008484

Smith, R., Lane, R. D., Parr, T., \& Friston, K. J. (2019). Neurocomputational mechanisms underlying emotional awareness: insights afforded by deep active inference and their potential clinical relevance. Neuroscience \& Biobehavioral Reviews, 107, 473-491. https://doi.org/10.1016/j.neubiorev.2019.09.002

Smith, R., Parr, T., \& Friston, K. J. (2019). Simulating emotions: An active inference model of emotional state inference and emotion concept learning. Frontiers in Psychology, 10, 2844. https://doi.org/10.3389/fpsyg.2019.02844

Smith, R., Schwartenbeck, P., Parr, T., \& Friston, K. J. (2020). An active inference approach to modeling structure learning: concept learning as an example case. Frontiers in Computational Neuroscience, 14, 41. https://doi.org/10.3389/fncom.2020.00041

Steiner, J. (1987). The interplay between pathological organizations and the paranoidschizoid and depressive positions. International Journal of Psycho-analysis, 68, 6980. PMID: 3570674

Steiner, J. (1992). The equilibrium between the paranoid-schizoid and the depressive positions. In Clinical lectures on Klein and Bion (pp. 46-58). Tavistock/Routledge.

Streufert, S., \& Streufert, S. C. (1969). Effects of conceptual structure, failure, and success on attribution of causality and interpersonal attitudes. Journal of Personality and Social Psychology, 11(2), 138. https://doi.org/10.1037/h0027047 
Target, M., \& Fonagy, P. (1996). Playing with reality: II. The development of psychic reality from a theoretical perspective. International Journal of Psycho-analysis, 77, 459-479. PMID: 8818764

Tenenbaum, J. B., \& Griffiths, T. L. (2001). Structure learning in human causal induction. In T. K. Leen, T. G. Dietterich, \& V. Tres (Eds.). Advances in neural information processing systems 13: Proceedings of the 2000 conference (pp. 59-65). MIT Press.

Tomov, M. S., Dorfman, H. M., \& Gershman, S. J. (2018). Neural computations underlying causal structure learning. Journal of Neuroscience, 38(32), 7143-7157. https://doi.org/10.1523/JNEUROSCI.3336-17.2018

Veen, G., \& Arntz, A. (2000). Multidimensional dichotomous thinking characterizes borderline personality disorder. Cognitive Therapy and Research, 24(1), 23-45. https://doi.org/10.1023/A:1005498824175

Wellstein, K., Diaconescu, A., Kasper, L., Bischof, M., Rüesch, A., Paolini, G., Mathys, C., \& Stephan, J. U. K. E. (2020). Linking subclinical persecutory ideation to inflexible social inference under uncertainty. Schizophrenia Bulletin, 46, S255-S256. https://doi.org/10.1093/schbul/sbaa029.624

Westen, D. (1991). Social cognition and object relations. Psychological Bulletin, 109(3), 429. https://doi.org/10.1037/0033-2909.109.3.429

Whitesell, N. R., \& Harter, S. (1989). Children's reports of conflict between simultaneous opposite-valence emotions. Child Development, 673-682. https://doi.org/10.2307/1130732

Widen, S. C., \& Russell, J. A. (2003). A closer look at preschoolers' freely produced labels for facial expressions. Developmental Psychology, 39(1), 114. https://doi.org/10.1037/0012-1649.39.1.114

Widen, S. C., \& Russell, J. A. (2008). Children acquire emotion categories gradually. Cognitive Development, 23(2), 291-312. https://doi.org/10.1016/j.cogdev.2008.01.002 
Winnicott, D. W. (1945). Primitive emotional development. International Journal of Psychoanalysis, 26, 137-143. PMID: 20984272

World Health Organization (1992). The ICD-10 classification of mental and behavioural disorders: clinical descriptions and diagnostic guidelines. World Health Organization.

World Health Organization. (2018). International classification of diseases for mortality and morbidity statistics (11th Revision). Retrieved from https://icd.who.int/browse11/1-en

Zanarini, M. C., Weingeroff, J. L., \& Frankenburg, F. R. (2009). Defense mechanisms associated with borderline personality disorder. Journal of Personality Disorders, 23(2), 113-121. https://doi.org/10.1521/pedi.2009.23.2.113

Zepf, S. (2012). Do we need the concept of "splitting" to understand borderline structures? The Scandinavian Psychoanalytic Review, 35(1), 45-57. https://doi.org/10.1080/01062301.2012.10592379

Zuckerman, M. (1979). Attribution of success and failure revisited, or: The motivational bias is alive and well in attribution theory. Journal of Personality, 47(2), 245-287. https://doi.org/10.1111/j.1467-6494.1979.tb00202.x 
